\title{
Abstracts of the 7th Symposium on Neuropsychological Rehabilitation Special Interest Group of the World Federation of NeuroRehabilitation July 5-6, 2010, Krakow
}

\section{Organising Committee}

Professor Barbara Wilson (Convenor), MRC Cognition \& Brain Sciences Unit and the Oliver Zangwill Centre, Cambridge, United Kingdom

Professor James Malec (Program Chair), Rehabilitation Hospital of Indianapolis and Department of Physical Medicine and Rehabilitation, Indiana University School of Medicine, Indianapolis, Indiana, United States of America

Dr Teresa A. Ashman, Rusk Institute of Rehabilitation Medicine, New York UniversityLangone Medical Center, New York, United States of America

Dr Andrew Bateman, Oliver Zangwill Centre, Princess of Wales Hospital Ely, United Kingdom

Dr Thomas Bodner, Universitätsklinik für Neurologie, Innsbruck, Austria

Dr Sara Cavaco, HGSA, Doenças Neurológicas e Órgãos dos Sentidos Largo, Porto,

Portugal

Professor Keith Cicerone, JFK-Johnson Rehabilitation Institute, Edison, United States of America

Dr Angela Colantonio, Saunderson Family Chair in Acquired Brain Injury Research, Toronto Rehabilitation Institute, University of Toronto, Toronto, Canada

Dr Fofi Constantinidou, University of Cyprus, Department of Psychology, Nicosia, Cypres

Professor Jonathan Evans, Section of Psychological Medicine, Faculty of Medicine,

University of Glasgow, Glasgow, United Kingdom

Dr Fergus Gracey, Oliver Zangwill Centre for Neuropsychological Rehabilitation, Ely, United Kingdom

Dr Steffen-Malik Hoegh, Vejlefjord - Specialized Rehabilitation Hospital, Stouby, Denmark

PD Dr. Reiner Kaschel, Neuropsychologische Praxis, Ebersbach/Fils, Germany

Dipl.-Psych. Herbert König, Akademie bei König \& Müller, Würzburg, Germany

Dr Dr Jan Lexell, Department of Clinical Sciences, Lund University, Lund, Sweden

Professor Emilia Lojek, Warsaw University, Pracownia Neuropsychologii Klinicznej Warsaw, Poland

Dipl.-Psych. Gerhard Müller, Akademie bei König \& Müller, Würzburg, Germany

Dr Tamara Ownsworth, School of Psychology, Griffith University, Australia

Professor Jennie Ponsford, Department of Psychology, Monash University, Australia

Dr Ronald T. Seel, Crawford Research Institute, Shepherd Center, Atlanta, United States of America

Professor Robyn Tate, Rehabilitation Studies Unit, Northern Clinical School, Sydney Medical School, University of Sydney, Australia

Professor Jean-Luc Truelle, Service de médecine physique et de réadaptation, C.H.U Raymond-Poincaré 92380 Garches, Paris, France

Dr Ann Watts, Entabeni Hospital, Durban, South Africa 


\title{
CONVENOR'S ADDRESS
}

\section{'It is not just the kind of head injury but the kind of head': Factors Influencing} Recovery from Traumatic Brain Injury

B.A. Wilson

MRC Cognition \& Brain Sciences Unit and the Oliver Zangwill Centre, United Kingdom

\begin{abstract}
This paper discusses contributing factors relating to recovery among 1 patients who have sustained a traumatic brain injury (TBI). As recovery means different things to different people, the term is used here to mean partial recovery of function together with substitution of function. Following on from a brief account of primary and secondary brain damage and natural changes which may be seen in people with TBI, consideration is given to factors influencing recovery, particularly age, gender and cognitive reserve. Age is only one factor in the recovery process that has to be considered alongside other, perhaps more important factors, such as (a) whether the lesion is focal or diffuse, (b) the severity of the insult, and (c) the time since acquisition of the function under consideration. There is conflicting evidence on the issue of whether females recover better than males. It would appear that those with higher intelligence or with better cognitive processing strategies may fare better than those without such cognitive reserve. Mechanisms of recovery are also examined, particularly regeneration, diaschisis and plasticity. The concepts of regeneration (re-growth of nerve cells), diaschisis (reduction of neural shock) and plasticity (anatomical reorganisation of the neural network) may all have a part to play in the mechanism of recovery. In conclusion, there is no doubt that considerable recovery can and does take place after TBI.
\end{abstract}

\section{SESSION 1: BRAIN REHABILITATION PROGRAMS}

\section{Investigation of a Brief Intervention to Minimise Alcohol Use Following Traumatic Brain Injury}

J. Ponsford ${ }^{1}$, L. Tweedly ${ }^{1}$ and N. Lee ${ }^{2}$

1 School of Psychology and Psychiatry, Monash University, Australia

2 Turning Point Alcohol and Drug Centre, Victoria, Australia

Background and aims: Pre-existing problems with alcohol abuse are common among survivors of traumatic brain injury (TBI) and continued alcohol consumption post-injury has been linked with poorer outcomes. Within the addiction literature, brief interventions based on a motivational style interview have been successful in reducing alcohol use. However there have been few such studies in TBI individuals. This study aimed to examine the relative effectiveness of a brief motivational interview (MI) combined with an information booklet, information booklet alone or treatment as usual in reducing alcohol consumption post-injury. Method: The study was a randomised controlled trial (RCT) with three groups. Sixty participants from Epworth Rehabilitation Centre, who had sustained a moderate to severe head injury 6 to 9 months previously, were randomly allocated into an MI intervention + information, information only, or treatment as usual group. At the initial assessment, information regarding alcohol and drug use, readiness to change behaviour, depression and anxiety, cognitive functioning, and functional status were collected. They were repeated at follow-up assessments six months later. Results: Preliminary results suggest that both interventions 
resulted in a reduction in the quantity and frequency of alcohol consumption relative to treatment as usual. Discussion and conclusions: The finding that such simple interventions can be useful in reducing alcohol use following TBI suggests that they should be introduced as standard practice and hopefully provide individuals with a better chance of optimal recovery and quality of life.

\title{
Effectiveness of Community-Based Rehabilitation After Traumatic Brain Injury (TBI) for 489 Program Completers Compared to Those Precipitously Discharged
}

\author{
I. Altman', S. Swick', D. Parrott ${ }^{2}$ and J. F. Malec ${ }^{2,3}$ \\ ${ }^{1}$ Rehabiltation Without Walls, Scottsdale, United States of America \\ ${ }^{2}$ Rehabilitation Hospital of Indiana, Indianapolis, United States of America \\ ${ }^{3}$ Rehabilitation Hospital of Indiana/Indiana University School of Medicine, Indianapolis, \\ United States of America
}

Rackground and aims: Because of the complexity of the intervention and B ethical concerns, postacute brain injury rehabilitation programs (PABIR) programs challenge rigorous experimental evaluation. Consequently, large observational trials are of value. This study examined outcomes of a homeand community-based PABIR conducted in 7 geographically distinct US cities. Method: Participants in this retrospective study included 489 consecutive admissions with traumatic brain injury (TBI) who completed the prescribed rehabilitation program (Completed course of Treatment; CCT group) and 114 discharged prior to program completion (Precipitous Discharge; PD group). Outcomes were assessed with the Mayo-Portland Adaptability Inventory (MPAI-4) completed by professional consensus on admission and at discharge. The MPAI-4 Participation Index only was obtained 3- and 12months after discharge through telephone contact. Rehabilitation services were provided in home and community settings by certified professional staff on an individualised basis. Results: Analysis of covariance (ANCOVA), with group (CCT vs. PD) as the between-subjects variable and MPAI-4 score at baseline as the covariate, showed significant differences between the groups at discharge for the full MPAI-4 $(F=82.25, p<.0001)$, the Abilities Index $(=50.24, p<.0001)$, the Adjustment Index $(F=81.20, p<.0001)$, and the Participation Index $(F=59.48, p<.0001)$. Despite a significant dropout rate, available data indicated that group differences remained statistically significant at 3- and 12-month follow-up. Discussion and conclusions: Results provide evidence of the effectiveness of community-based rehabilitation and that treatment effects are maintained at follow-up.

\section{Therapeutic Alliance of Significant Others With the Staff of a Holistic Day Program: An Important Facilitator of the Successful Response of Patients With Traumatic Brain Injury (TBI) to Neuropsychological Rehabilitative Interventions}

\author{
E. Daniels-Zide and Y. Ben-Yishay \\ New York University School of Medicine, NYU Langone Medical Center, Rusk Institute, Brain Injury Day \\ Treatment Program, New York, United States of America
}

recent pilot study that was undertaken in the setting of the NYU-Rusk
of neurtitute intensive, holistic ('therapeutic community'-type) day program
who proved to be most successful in responding to the program's philosophy
and to its remedial and therapeutic interventions, shared in common certain
premorbid temperamental and personality traits. Success was measured by a
13-item multidimensional, weighted scale scoring system. (Successful 
Rehabilitant Index, SREI; Cronbach's $\alpha=.987$ ). Common (presumed) premorbid personality traits were identified by the raw scores on the 30 'phasics' (subscales) of the standardised NEO personality Inventory Test. Statistical analyses revealed that: (a) of the 30 NEO 'phasics', 16 correlated (at $p<.01$ ) with SREI; (b) $t$ tests for the differences between the mean NEO raw scores of the top 15 successful rehabilitants versus the bottom 15 rehabilitants were also found to be significant (at $p<.01$ ); and (c) multivariate regression analyses, to predict the successful rehabilitant index, by using only three of the NEO phasics (competence, $r=.653$; Positive Emotions, $r=.633$; Self-Discipline, $r=$ .620) accounted for $64 \%$ of the total amount of variance. The present pilot study is a follow-up on the preceding study. It sought to support previous findings that a therapeutic alliance between the significant others (SO) of TBI program trainees' and the staff, facilitated the successful responses of the TBI trainees to rehabilitative interventions. The degree of the Therapeutic Alliance between the SO and the staff was measured by an 8-item multidimensional, cumulative weighted scale scoring system (Significant Others' Therapeutic Alliance, SOTA; Cronbach's $\alpha=.951$ ). This was done in two steps. Step One: 15 randomly selected SO were independently rated by the staff with inter-rater reliabilities ranging from .871 to .946 . Then, all $32 \mathrm{SO}$ (4 SO from the preceding study could attend the program only on rare occasions!) were rated by the clinical staff based on clinical consensus. Preliminary statistical analyses revealed (a) that successful rehabilitant index, SRSEI, correlated highly ( $r=$ .569) with significant others' therapeutic alliance scores, SOTA, and (b) multivariate regression analyses, aimed at predicting the successful rehabilitant index, in which only three predictor variables were used (NEO Positive emotions; NEO Achievement Striving and SOTA) yielded a multiple R of .781, or accounted for $61 \%$ of the total amount of variance. Conclusions, additional studies, and implications for treatment will be briefly discussed.

\section{Social Cognition Rehabilitation in Veterans With Traumatic Brain Injury (TBI) and Posttraumatic Stress Disorder (PTSD)}

C. Campbell ${ }^{1}$, K. McCoy ${ }^{2}$ and M. Kelly ${ }^{3}$

${ }^{1}$ Polytrauma/Psychology, Veterans Affairs Medical Center, Washington, DC, United States of America

2 War-Related Injury \& IIIness Study Center, Veterans Affairs Medical Center, Washington, DC, United States of America

${ }^{3}$ Speech, Language \& Audiology, Veterans Affairs Medical Center, Washington, DC, United States of America

$\boldsymbol{B}$ ackground: Psychosocial functioning plays a significant role in both read$\boldsymbol{B}_{\text {justment after traumatic brain injury (TBI) and in recovery from post-trau- }}$ matic stress disorder (PTSD). Effective social interaction relies on the successful application of numerous cognitive functions that are prone to disruption by TBI and PTSD, including: emotion perception in self and others, social problem solving, implementation of intentions, evaluation of outcomes and motivation. There are no known treatments designed for individuals with both TBI and PTSD, despite this common comorbidity, and existing treatments do not address the complex factors involved in successful social reintegration. Methods: Clinicians at the Washington, DC Veterans Affairs Medical Center have developed a prototype social cognition group for veterans with comorbid TBI and PTSD that is anchored in existing research and firsthand clinical knowledge. The group is divided into three interrelated modules aimed to: (1) improve emotion perception in self and others, (2) enhance social problem solving, and (3) encourage identity development and social readjustment. Intervention 
techniques include didactics, discussion, practice, videotaping and in vivo experiential learning. Results: This presentation will (1) Review the research informing the theoretical basis for the social cognition group; (2) Describe the curriculum for the group; (3) Demonstrate specific interventions used within the group; and (4) Present individual case examples of the benefits derived from the group. Discussion/Conclusions: For individuals with overlapping cognitive and emotional symptoms, an integrated neuropsychotherapy approach holds promise for improving social interaction abilities and satisfaction. Implications and plans for further research will also be addressed.

\title{
SESSION 2: Methods and Measurement
}

\section{What Quality of Life After Traumatic Brain Injury? QOLIBRI, a Novel Disease-Specific Quality of Life Measure}

\author{
J.-L. Truelle ${ }^{1}$, K. von Wild ${ }^{2}$ and N. von Steinbüchel ${ }^{3}$ \\ ${ }^{1}$ Neurorehabilitation Department, University Hospital, Garches, France \\ 2 kvw-neuroscience consulting GmbH, Münster, Germany \\ ${ }^{3}$ Medical Psychology, Georg-August-University, Göttingen, Germany
}

\begin{abstract}
Qbjective: To present the QOLIBRI, a disease-specific measure of quality of life after traumatic brain injury (TBI). Methods: The QOLIBRI, with 37 items in six scales (cognition, self, daily life and autonomy, social relationships, emotions, and physical problems) was completed by 795 patients in six languages (Finnish, German, Italian, French, English and Dutch). QOLIBRI scores were examined by socio-demographic variables, disability recovery, health status and mental health. Results: The QOLIBRI was self-completed by $73 \%$ of participants in 11 minutes, and $27 \%$ completed it in interview in 20 minutes. It was sensitive to areas of life amenable to intervention, such as living arrangement, work, health status (including mental health), and functional outcome. Conclusion: The QOLIBRI provides information about a patient's subjective perception of his/her quality of life which supplements clinical measures and measures of functional outcome. It can be applied across different populations and cultures. It allows the identification of personal needs, the prioritisation of therapeutic goals, and the evaluation of individual progress. It may be useful in clinical trials and in longitudinal studies of TBI recovery.
\end{abstract}

\section{Assessing Change in Executive Functioning Using a Rasch Modelling Approach}

\author{
A. Bateman ${ }^{1}$ and S. Simblett ${ }^{2}$ \\ ${ }^{1}$ Oliver Zangwill Centre for Neuropsychological Rehabilitation, Cambridgeshire Community Services, \\ The Princess of Wales Hospital, Ely, United Kingdom \\ 2 Psychiatry, University of Cambridge, Cambridge, United Kingdom
}

The Oliver Zangwill Centre provides an intensive holistic neuropsycho1 logical rehabilitation program in Ely, UK. Clients address a range of cognitive, emotional and vocational rehabilitation goals through attendance in groups and one-to-one therapy sessions with Occupational Therapists, Speech and Language Therapists and Clinical Psychologists. Executive functions are addressed by the whole team in relation to the client's personal goals as well as generically in 'Cognitive Group'. A measure of executive functioning, the dysexecutive (DEX) questionnaire, is administered to all clients and their carers before and after rehabilitation. To explore change in executive functions, 75 client's self-ratings on the DEX questionnaire were 
analysed using newly emerging techniques grounded in a Rasch modelling approach. Using new subscales that emerged from a previous analysis on a larger sample of 335, differential item functioning between time-points was assessed to check for item bias, and person-location values were analysed to evaluate response to rehabilitation. There was no item bias, suggesting that items display similar meanings prior to and following rehabilitation, enabling valid comparisons to be made between responses. The average reduction in the probability of endorsing items of the DEX questionnaire ranged between 0.11 and 0.14 for all subscales after rehabilitation. This approach to analysis of reassessment data represents an important step, as objective measurement theories are often overlooked in intervention studies. Change as measured on an interval scale in this way enables us to now examine the factors mediating change using further parametric techniques with a view to refining our interventions with greater precision.

\title{
Statistical Analysis for Single Case Experimental Design Research Studies in Neuropsychological Rehabilitation: Controversies, Recent Developments and Some Recommendations
}

\section{J. Evans}

\section{Section of Psychological Medicine, University of Glasgow, Glasgow, United Kingdom}

\begin{abstract}
$\boldsymbol{R}$ ackground and aims: Single case experimental designs provide a useful methodology for measuring the effectiveness of neuropsychological rehabilitation interventions. The recently published Single Case Experimental Design (SCED) Scale (Tate et al., 2008) provides a means of evaluating the methodological quality of single-N studies and aids research planning. One limitation of the SCED Scale is that it does not specify which approaches to statistical analysis of SCED data are most appropriate. Indeed, the question of whether statistical analysis is necessary in SCEDs and, if so, which methods are appropriate remains controversial. Many different statistical techniques have been proposed but no consensus has emerged. The aim of this paper is to identify the analysis methods proposed for SCED data, review recent advances and make recommendations regarding appropriate methods. Method: A systematic search of the research literature relating to the evaluation of methods of statistical analysis for SCED data was undertaken. The aim was to identify methods that (a) are appropriate for short autocorrelated data series, (b) provide a measure of effect size, (c) are simple to compute. The performance of a wide range of analysis techniques were collated and reviewed in relation to these criteria. Results: The methods that meet all three criteria are variations of the 'Percentage of Nonoverlapping Data' approach, including several recent adaptations designed to remedy weaknesses in traditional methods. Discussion and conclusions: To maximise the contribution of SCED studies to evidence-based practice in neuropsychological rehabilitation, consistent use of methods that facilitate collation, comparison and meta-analysis of data is required.
\end{abstract}




\title{
Predicting Duration of Posttraumatic Amnesia (PTA) Across the Spectrum of Traumatic Brain Injury (TBI) Severity: Can it be Done?
}

\author{
M. Perdices ${ }^{1,2}$, R. L. Tate ${ }^{3}$, A. Pfaff ${ }^{4}$, G. Herkes ${ }^{1,2}$, A. Hodgkinson ${ }^{4}$, P. David ${ }^{3}$ \\ and A. Cassel ${ }^{3}$ \\ ${ }^{1}$ Department of Neurology, Royal North Shore Hospital, Sydney, Australia \\ ${ }^{2}$ Northern Clinical School, Sydney Medical School, University of Sydney, Sydney, Australia \\ ${ }^{3}$ Rehabilitation Studies Unit, Northern Clinical School, Sydney Medical School, University of Sydney, \\ Sydney, Australia \\ ${ }^{4}$ Brain Injury Rehabilitation Unit, Liverpool Hospital, Sydney, Australia
}

\begin{abstract}
Rackground: A challenge in the post-acute rehabilitation of patients with $\boldsymbol{D}_{\mathrm{TBI}}$ is being able to anticipate when they will emerge from PTA. Using regression analysis, we have previously developed predictive models to estimate duration of PTA in patients with very severe TBI (PTA $>1$ month). In a sample of 61 patients, day post-trauma PTA testing commenced and scores on the Modified Oxford PTA Scale for the first five days of testing, accurately predicted duration of PTA, accounting for $88 \%$ of the variance (Tate et al., 2001). In a subsequent sample $(n=84)$ patients, scores for the first three days of testing accurately predicted duration of PTA, accounting for $45 \%$ of the variance (Tate et al., 2006). The present study investigated the predictive value of the second model in patients with milder TBI. Method: Fifty-five patients were tested during PTA (mean PTA duration $=10.1$ days). Regression analyses were conducted using the summed score for the first three days of PTA testing. Results: A model accounting for $74 \%$ of the variance was derived. Correlation between observed and predicted days to emergence from PTA was $.86(p<.0001)$. The sample was then combined with the original group $(n=84)$. Mean duration of PTA in the combined group $(n$ =139) was 30.7 days (range 1-88). A model accounting for $54 \%$ of the variance was derived. Correlation between observed and predicted days to emergence of PTA was .74 $(p<.0001)$. Conclusion: Duration of PTA in patients with TBI can be reliably predicted across the spectrum of TBI severity.
\end{abstract}

\section{SESSION 3: DATABLITZ - CLINICAL ASSESSMENT}

\section{Neuropsychological Assessment of the Locked-in-Syndrome: Challenges,} Consequences and Cognitive Functioning

\footnotetext{
B. Wilson ${ }^{1}$, A. Hinchcliffe ${ }^{2}$ and G. Florschutz ${ }^{2}$

${ }^{1}$ MRC Cognition \& Brain Sciences Unit and the Oliver Zangwill Centre, United Kingdom ${ }^{2}$ Speech and Language Therapy, Raphael Medical Centre Raphael Medical Centre, Kent, United Kingdom
}

Rackground: Locked-in-Syndrome (LIS) is a rare consequence of brain

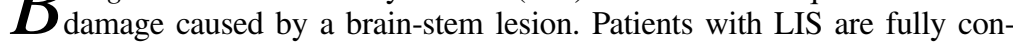
scious but unable to move or speak due to paralysis of nearly all voluntary muscles except the eyes. The best known case is probably that of JeanDominique Bauby who 'wrote' The Diving Bell and the Butterfly. We present a young woman, VQ, who survived a mid-brain stroke resulting in the LIS. Aims: Although patients with the LIS are reported to be cognitively intact, formal neuropsychological assessments are rarely documented. One exception is Smart et al. (2008). Our aims were to: (a) describe the challenges faced in the assessment of LIS; (b) consider the most appropriate tests for them; and (c) determine if our patient had normal cognitive functioning. Methods: VQ communicates by raising her eyes for 'yes' and lowering them for 'no'. Those 
communicating with her use a communication board to present letters and numbers. Standardised tests of reading (lexical decision), naming, memory, perception and problem solving were administered. Although VQ communicates quickly, her communication is slower than speech and certain tests cannot be given. Consequently modifications have to be made to test administration. Results: VQ's reading, naming and problem solving scores are in the good average-superior range. She appears to have some mild impairment with tests of recognition memory. Conclusions: (1) With some planning and adjustment, it is possible to formally assess a patient with LIS; (2) Although cognitive functioning in this patient was, for most domains, average or above, she would appear to have some mild difficulties with recognition memory.

\title{
Predictive and Mediating Factors of Autobiographical Memory Retrieval Following Acquired Brain Injury
}

\author{
A. Duckworth', H. Williams ${ }^{1}$, M. Bunnage ${ }^{2}$ and L. Mounce ${ }^{1}$ \\ ${ }^{1}$ School of Psychology, University of Exeter, Exeter, United Kingdom \\ 2 Neuropsychology, North Bristol NHS Trust, Bristol, United Kingdom
}

\begin{abstract}
$\boldsymbol{B}$ ackground: Autobiographical memory (AM) deficits are a common type $\boldsymbol{B}_{\text {of cognitive difficulty after acquired brain injury (ABI). AM is pivotal }}$ for everyday functioning and a continued sense of identity. Emotional difficulties are also known to affect AM ability. We aimed to: explore the contribution of neurological, cognitive and affective factors on AM retrieval following acquired brain injury. Methods: A cross-sectional, within-subjects design. Participants with acquired brain injury were recruited through Frenchay Hospital, Bristol and Headway. Participants were 18 years and over, and not with severe mental health, alcohol/drug or executive disorders. Data was obtained from traditional neuropsychological assessments of memory, executive and attention abilities. Measures of mood, post-traumatic stress, rumination and of autobiographical specificity were given. Results: Data will be presented regarding regression analysis on variance of each of the predictor variables as they relate to AM specificity. Conclusions: AM can be compromised by neurological and psychological factors. A better understanding of how these may converge to reduce AM specificity in acquired brain injury may indicate how neuro-rehabilitation can be improved to enable better access to personal memories and improve mood.
\end{abstract}

\section{Dimensions of the Dysexecutive Questionnaire (DEX) Examined Using Rasch Analysis}

\author{
S. Simblett ${ }^{1}$ and A. Bateman ${ }^{2}$ \\ ${ }^{1}$ Psychiatry, University of Cambridge, Cambridge, United Kingdom \\ 2 Oliver Zangwill Centre for Neuropsychological Rehabilitation, Ely, United Kingdom
}

Changes in the ability to plan, problem-solve, make decisions, initiate actions, multitask, and regulate emotions are frequently encountered after acquired brain injury (ABI). These executive functions are thought to all contribute to the ability to adapt and respond appropriately to the environment, as well as prioritise and maintain focus on goals. Many argue that the concept of executive functions should be subdivided into smaller components. In this study we explored the dimensional structure of a tool designed to assess executive functions, the DEX questionnaire (Wilson et al., 1996). Rasch analysis using the software package RUMM2020 was completed on the responses of 363 clients assessed at the Oliver Zangwill Centre for 
Neuropsychological Rehabilitation. Items relating to problems with distractibility, planning, decision making and abstract thinking were more frequently endorsed among our sample. Overall the scale did not perform as expected by the Rasch model (with significant item-trait misfit Chi-square $=$ $122.67, p<.001$ ). Of particular interest was that none of the subscales proposed in the original DEX Manual met all the assumptions of the Rasch model, suggesting that they do not function as measures of executive functioning in their original form. Several new subscales are proposed, alongside suggestions for revision of the wording and scoring of some of the items in the DEX questionnaire. These results provide a platform for future evaluation of rehabilitation programs with a focus on executive functions.

\title{
Differentiating Between Spontaneous Recovery and Treatment-Specific Effects in the Postacute Phase After Traumatic Brain Injury
}

\author{
R.L. Tate ${ }^{1}$, C. Taylor ${ }^{2}$, M. Perdices ${ }^{3}$, V. Aird ${ }^{2}$ and A. McCarry ${ }^{2}$ \\ ${ }^{1}$ Rehabilitation Studies Unit, Northern Clinical School, Sydney Medical School, \\ University of Sydney, Australia \\ ${ }^{2}$ Brain Injury Rehabilitation Unit, Royal Rehabilitation Centre Sydney, Sydney, Australia \\ ${ }^{3}$ Department of Neurology, Royal North Shore Hospital, Sydney, Australia
}

Rackground: In the initial months following severe traumatic brain injury $\boldsymbol{B}$ (TBI) functional improvement is generally attributed to spontaneous recovery. In many treatment facilities, inpatient rehabilitation also occurs in this critical timeframe. The question thus arises as to whether improvement is due to intervention provided by the therapist/s, spontaneous recovery of brain function, or a combination of both. In the present paper we present a single-case experimental design within the context of a new model of clinical practice (Tate, Taylor, Aird \& McCarry, 2010). Method: A 26 year-old male sustained severe TBI and on admission to inpatient rehabilitation Functional Independence Measure score was 22 (Motor 17; Cognitive 5). After emergence from post-traumatic amnesia two months following admission, a 10-week intervention was developed to treat expressive language deficits, aiming to increase utterance length, using a multiple baseline design across behaviours. Three tasks were simultaneously measured but sequentially treated: picture description, story recall and topic-centred narrative. Stimuli assessing the target behaviours were divided into two sets: measures using the treated stimuli were taken at the commencement of each (baseline and therapy) session; probes using untreated stimuli were taken at six points during the 20-session intervention. Results: The three tasks showed different responses to treatment, with picture description demonstrating the clearest effect of treatment in comparison with baseline $(z=-3.17, p=.001)$. Story recall showed rising baseline that continued into the therapy period and was considered to mainly reflect spontaneous recovery of memory function rather than language-specific effects of therapy, although use of untreated probes also provided evidence of specific treatment effect that went above and beyond spontaneous recovery. Conclusions: Use of the multiple baseline design with untreated probes provides a model to enable a distinction to be made between spontaneous recovery and improvements specifically due to the therapy intervention. 


\title{
Using the Wessex Head Injury Matrix (WHIM) for Goal Setting in Minimally Conscious States
}

\author{
M. Darcy ${ }^{1}$ and J. Tierney ${ }^{2}$
}

${ }^{1}$ Private Physiotherapy Practice, North Fitzroy, Australia

2 Brain Disorders Program, Austin Health, Kew, Australia

\begin{abstract}
Tntroduction: There is often a nihilistic therapeutic approach to patients who are in a Minimally Conscious State (MCS) for prolonged periods of time. There are very few reports of therapeutic interventions to enhance outcomes in MCS. Aim: To explore the use of the Wessex Head Injury Matrix as a tool in therapeutic goal setting in MCS. Method: Case studies will be used to describe a method incorporating goal setting using the WHIM, establishing therapeutic interventions based around these goals, and the implementation of these interventions within functional activities of the persons' usual daily care programs. Target behaviours were recorded with 24 hour charting for 1 week before and after the intervention period. WHIM scores were recorded before and after the intervention period. Individual daily care programs had previously been established to ensure all manual handling, positioning, and personal care interventions were below startle threshold. Comfort was maintained throughout 24 hours of the day, and gross movement patterns minimised. Results: Goal setting using the WHIM enabled the development of additional functional skills to establish intentional movement and an increased level of participation in daily care. Discussion: MCS does not preclude participation in a rehabilitation program based around everyday activities.
\end{abstract}

\section{SESSION 4: PEDIATRIC ISSUES}

\section{Peer-Relationship Difficulties in Children With Brain Injuries: Comparisons With Children in Mental Health Services and Mainstream Schools}

\author{
J. Tonks ${ }^{1}$, W. H. Williams ${ }^{1}$, I. Frampton ${ }^{2}$, P. Yates ${ }^{3}$ and A. Slater ${ }^{1}$ \\ ${ }^{1}$ School of Psychology, University of Exeter, Exeter, United Kingdom \\ 2 Cornwall Partnership NHS Trust, Cornwall, United Kingdom \\ ${ }^{3}$ Royal Devon and Exeter Hospital, Exeter, United Kingdom
}

\begin{abstract}
As $s$ children with traumatic brain injury (TBI) become adolescents, they struggle to keep up with rapidly developing same age peers. They are less adept at recognising and responding to expressions; more sophisticated social situations may become too challenging and complex; and there may be considerable differential development in reaching cognitive/intellectual milestones. Difficulties typically increase over time, and children with TBI are at increased risk of later-life clinically significant difficulties. Aims: In this study we wanted to explore the prevalence of peer difficulties in TBI children compared to those reported by a matched, non-injured clinical sample of children accessing mental health services and a non-injured, nonclinical sample. Methodology: 39 TBI children, 57 children from the Child and Adolescent Mental Health Service (CAMHS) and 67 children in mainstream schools participated in the study. Parents, teachers and children were asked to complete a measure of peer-relationship difficulties. Results: CAMHS children and TBI children experienced significantly more peerrelationship difficulties than controls. There was no significant difference between CAMHS children and TBI children in terms of the level of peer-relationship difficulties reported. Conclusions: TBI children are as
\end{abstract}


disadvantaged as children with mental health difficulties in relationships with peers, yet appropriate intervention services are not commonly available after TBI. Improved provision for TBI children with peer-relationship difficulties may result in more positive outcomes for such children in adulthood.

\title{
A Systematic Review of Ecological Measures of Cognitive Functioning for Children With Acquired Brain Injury
}

\author{
M. Chevignard ${ }^{1}$, C. Soo ${ }^{2}$, J. Galvin ${ }^{3}$ and S. Eren ${ }^{2}$ \\ ${ }^{1}$ Rehabilitation of children with acquired brain injuries, Hospital National de Saint Maurice, \\ Saint Maurice, France \\ ${ }^{2}$ Australian Centre for Child Neuropsychology Studies, Murdoch Children's Research Institute, \\ Victoria, Australia \\ ${ }^{3}$ Victorian Paediatric Rehabilitation Service, Royal Children's Hospital, Victoria, Australia
}

\begin{abstract}
$B$ ackground and aims: Acquired brain injury (ABI) sustained in childhood often leads to impairment in cognitive functioning. These deficits may be responsible for longstanding disabilities in a child's life both at home and school. The ability of traditional neuropsychological tests to accurately measure cognitive abilities as they present in everyday life has been questioned. This study aims to systematically review ecological measures of cognitive abilities available for children with ABI. Method: Eight data-bases were searched (until April 2008) for scales (1) focused on ecological assessment of cognitive functioning; (2) with published data in an ABI population; (3) available for children up to age 18 and (4) in English. The title and abstract of all papers were inspected independently by two reviewers. Results: Database searches yielded a total of 12,000 references, of which 11 scales met the inclusion criteria for the review, focusing on memory (Rivermead Behavioural Memory Test, Contextual Memory Test, Observer Memory Questionnaire), executive functions (EF; Behavioral Rating Inventory of Executive Function, Behavioural Assessment of Dysexecutive Syndrome Test Battery for Children, Party Planning Task); general cognitive abilities (Dynamic Occupational Therapy Cognitive Assessment for Children; Cognitive Failures Questionnaire), visuo-spatial skills (Kiel Locomotor Maze, Kicking test) and attention (Test of Everyday Attention for Children). Discussion and conclusions: Overall, few measures were found; four were experimental tasks; four were office-based. Some areas of cognitive function, like memory and EF, were better represented in ecological assessment than others, with relatively more standardised scales found, as well as more studies on these scales.
\end{abstract}

\section{Paediatric Traumatic Brain Injury: Siblings Outcome}

M. Sambuco' ${ }^{1}$ S. Lah', N. Brookes ${ }^{2}$ and C. Catroppa ${ }^{3}$

${ }^{1}$ School of Psychology, The University of Sydney, Australia

${ }^{2}$ Brain Injury Rehabilitation Program, Sydney Children's Hospital (Randwick), Randwick, Australia

${ }^{3}$ Department of Psychology, Australian Centre for Child Neuropsychology Studies (ACCNS), Murdoch Children's Research Institute, Royal Children's Hospital, Victoria, Australia

Rackground and aims: Children with traumatic brain injury (TBI) are at $\boldsymbol{B}_{\text {risk of residual deficits which have a significant impact on family func- }}$ tioning. Siblings of children that sustained TBI are likely to be affected by changes in family functioning. Nevertheless, minimal research exists on sibling outcomes. This study aims to extend understanding of sibling adjustment and identify variables that best predict sibling behavioural outcomes post pae- 
diatric TBI. It was anticipated that in addition to TBI specific factors, variables from a generic chronic illness model of sibling adjustment will also contribute to sibling behavioural outcome. Method: 39 siblings, --18 years, closest in age to a child who sustained moderate to severe TBI over 1 year ago participated. Self and parent questionnaires were used to assess sibling, parent, family, and injured child variables. Results: Sibling results indicated a markedly reduced sense of self-esteem and perceived lack of social support, but no increase in behavioural problems. Sibling behavioural outcomes were best predicted by a combination of generic (sibling knowledge of TBI, sibling sense of social support, parent mood) and specific (TBI child behaviour) variables. Discussion and conclusions: Our study confirmed that models arising from child chronic illness literature may be of relevance for sibling adjustment post TBI and identified factors associated with good behavioural outcome. Future studies should utilise knowledge from chronic illness literature to extend our understanding of reduced self-esteem and perceived lack of social support and develop programs to ameliorate these difficulties.

\section{Support Needs After Childhood Acquired Brain Injury: Disentangling Developmental and Brain Injury Issues}

C. Soo ${ }^{1}$, R. L. Tate ${ }^{2}$, V. Anderson' ${ }^{1}$ and M.-C. Waugh ${ }^{3}$

${ }^{1}$ Australian Centre for Child Neuropsychology Studies, Murdoch Childrens Research Insitute, Parkville, Australia

${ }^{2}$ Rehabilitation Studies Unit, Northern Clinical School, Sydney Medical School, University of Sydney, Sydney, Australia

${ }^{3}$ Brain Injury Rehabilitation Program, Children's Hospital at Westmead, Westmead, Australia

Rackground and aims: Children with acquired brain injury (ABI) may $\boldsymbol{B}_{\text {require support needs for a range of activities such as bathing, use of }}$ transport and social participation. One of the barriers to assessing such needs, however, is the lack of information on support needs of healthy children. This study aimed to provide normative data on support needs for a range of daily activities using the Paediatric Care and Needs Scale (PCANS). Methods: Participants were 300 parents/caregivers of healthy children (5-14 years) recruited from schools in Melbourne, Australia. Thirty children in 10 age-bands with approximately equal sex ratio were recruited. PCANS items were ordered according to the proportion in each age-band able to complete the activity independently. Results: Across the total sample, lower support needs were associated with increasing child age $(p<.01)$, with more children requiring supervision versus physical assistance for most PCANS domains $(p<.01)$. Across the age-bands, 33-74\% and $43-87 \%$ of children were found to be independent (i.e., did not require supports) for Shopping and use of Everyday Devices respectively. In contrast, few children were independent for Home Activities (0-7\%). The majority of items conformed to an expected hierarchy, but some items (e.g., 'taking regular medications', 'making health appointments') required reordering. Discussion and conclusions: Results of this study will allow refinement of item ordering of the PCANS providing unique baseline data for assessing the support needs related to childhood ABI. Findings will provide clinicians with a tool for disentangling developmental and brain injury issues when assessing support needs of children with ABI. 


\title{
SESSION 5: COGNITIVE REHABILITATION
}

\section{Improvements in Executive Functioning and Processing Speed After Cognitive} Rehabilitation With REHACOP: Schizophrenia and First-Episode Psychosis

\author{
E. Bengoetxea ${ }^{1}$, J. Peña ${ }^{1}$, N. Ojeda ${ }^{1}$, R. Segarr², P.M. Sanchez ${ }^{3}$, E. Elizagarate ${ }^{3}$, \\ J. Garcia ${ }^{2}$, J.I. Eguiluz ${ }^{2}$, J. Ezcurra ${ }^{3}$ and M. Gutierrez ${ }^{4}$ \\ ${ }^{1}$ Psicología y Educación, Universidad de Deusto, Bilbao, Spain \\ 2 Servicio de Psiquiatría, Hospital de Cruces, Barakaldo, Spain \\ ${ }^{3}$ Unidad de Psicosis Refractarea, Hospital Psiquiátrico de Álava, Spain \\ ${ }^{4}$ Hospital Santiago de Vitoria, Spain
}

\begin{abstract}
bjectives: Processing speed and executive functioning are among the more impaired cognitive domains in schizophrenia, do not improve despite antipsychotic medication, and are associated with poor long-term functioning and quality of life. Cognitive remediation therapy for psychosis (REHACOP) aims to improve cognitive deficits by teaching information processing strategies through guided mental exercises. The objective of this study is to evaluate the effectiveness of REHACOP, compared to other treatments, on improving processing speed and executive functioning difficulties. Material and Methods: Fifty-seven patients with DSM-IV schizophrenia and 29 with first-episode psychosis were randomly allocated into one of two groups: Cognitive rehabilitation group (REHACOP) or occupational therapy group. The REHACOP group received 3 months of structured group rehabilitation sessions ( 3 per week) focused on tasks requiring attention, language, memory, speed, executive functioning and activities of daily living. All subjects underwent a neuropsychological assessment pre- and post-treatment, which included tests for processing speed (Trail-Making Test-A, Digit Symbol, and Stroop-Color) and executive functioning (Stroop Word-Color part and interference). Results: Repeated measures of MANOVA showed that the interaction term (group $\mathrm{X}$ time) was significant for the executive functioning $(F=9.88, p<.01)$ and processing speed $(F=5.92, p<.05)$ measures, suggesting that the REHACOP experimental group improved significantly when compared to the control group in both domains.
\end{abstract}

\section{Investigating the Effect of Periodic Auditory Alerts in Conjunction With Goal Management Training on a Complex Virtual Reality Task in Individuals With Acquired Brain Injury}

\author{
P. Brown', R. Morris ${ }^{2}$ and J. Evans ${ }^{3}$ \\ ${ }^{1}$ Psychology Addictions Service, Glasgow, United Kingdom \\ 2 Department of Psychiatry, Institute of Psychiatry, London, United Kingdom \\ ${ }^{3}$ Section of Psychological Medicine, University of Glasgow, Glasgow, United Kingdom
}

$\boldsymbol{B}_{\text {ackground and aims: Deficits in planning and prospective memory are }}$ $\boldsymbol{B}$ common after brain injury and contribute to difficulties participating in everyday activities. Recent research has suggested that using non-contingent auditory alerts may facilitate a 'goal-review' process and improve performance on complex tasks that make demands on executive functions. This study investigated whether combining alerts with brief goal management training (GMT) would improve performance on a virtual reality task. Method: Twenty individuals with executive impairment completed two versions of the Removals Task, a virtual reality (VR) test of executive functions including prospective remembering. One trial was done with auditory alerts plus GMT, and the other in standard, non-alerted conditions. Nineteen healthy controls 
completed the task with no alerts or GMT. Results: The brain injury group was significantly poorer than controls on some, but not all, task measures in nonalerted conditions. GMT plus auditory alerts did not improve performance at a group level, though further analyses revealed improvement for a subgroup of the most impaired participants on one measure. Discussion and conclusions: It is important to understand conditions under which interventions work and don't work. While a subgroup of participants did show improvement in the alerted condition for one measure, GMT plus auditory alerts failed to improve performance in the brain injury group on the majority of task measures. Ceiling effects on some measures, brevity of the GMT procedure and paradoxical effects of the alerts on performance are discussed as some possible reasons for failure of the intervention to improve performance in the VR environment.

\title{
Memory Rehabilitation Following Stroke, Multiple Sclerosis, and Traumatic Brain Injury: A Mixed-Methods Randomised Controlled Trial
}

R. das Nair and N. Lincoln

Institute of Work, Health \& Organisations, University of Nottingham, Nottingham, United Kingdom

\begin{abstract}
Rackground: Memory problems are common following traumatic brain injury (TBI), stroke, and multiple sclerosis (MS). Systematic reviews of the effectiveness of memory rehabilitation indicate that the evidence is inconclusive, due to a paucity of well-conducted randomised controlled trials (RCTs). Objectives: To compare the effectiveness of a two types of memory rehabilitation programs with a control group. Methods: In this single blind RCT, 72 participants with memory problems following TBI, stroke, or MS were randomly allocated to one of two treatment programs (compensation or restitution) or a control (self-help) group by an independent agency. Memory functions, mood, and activities of daily living (ADL) were assessed at baseline. All programs were manual-based and comprised two individual sessions and 10 weekly group sessions, lasting 1.5 hours each. Blind follow-up assessments were conducted 5 and 7 months postrandomisation. Feedback interviews were audio-recorded and thematically analysed. Results: The intervention groups used significantly more internal memory aids than the control group, $F(2,69)=.49, p=.01$. There were also improvements in treatment groups but not in the control group on the Everyday Memory Questionnaire, but this did not reach statistical significance, $F(2,64)=.49, p=.61)$. No statistically significant differences over time or between groups were observed on measures of external memory aids use, mood or ADL. However, feedback interviews suggested added benefits in several domains. Conclusions: There was some evidence for effectiveness of memory rehabilitation in improving everyday memory either immediately post-intervention or at longer term follow-up. Small sample size and lack of sensitive assessment tools may have affected outcomes.
\end{abstract}

\section{Factors Influencing the Uptake of Memory Compensations: A Qualitative Analysis}

\section{Baldwin ${ }^{1}$ and T. Powell ${ }^{2}$}

${ }^{1}$ School of Psychology, University of Birmingham, Birmingham, United Kingdom

${ }^{2}$ Clinical Psychology, University of Birmingham, Birmingham, United Kingdom

Rackground and aims: In 1996 Wilson and Watson stated that 'there is B little general agreement or understanding concerning the reasons why some memory impaired people learn to use compensations efficiently and 
others fail to use them'. Yet there has still been no in-depth exploration of what motivates people to use memory compensations from the perspective of the individual with ABI. This qualitative study aims to appraise this gap in current knowledge in order to help therapists promote the use of memory compensations more effectively. Method: Eight people attending outpatient brain injury rehabilitation were interviewed. Transcripts were analysed using Interpretative Phenomenological Analysis. Results: Five master themes emerged from the analysis: (1) things that make it difficult to accept the use of strategies including factors that may have a reverse effect on motivation such as the strategy being an unpleasant reminder that one is different; (2) beliefs about memory, for example, it should be used or it will become lazy; (3) 'It's not in my nature', that is, it doesn't fit with the person's lifestyle; (4) the need for back up strategies to support the main strategy; and (5) characteristics of memory aids, for example, how they look and how effective they are at delivering cues that are timely. Discussion and conclusions: Previous quantitative studies have focused on factors such as type and extent of cognitive problems, age, and premorbid use of aids. This study suggests that motivation for strategy use depends on more complex processes that include social, emotional, and practical factors, all of which need to be considered.

\title{
SESSION 6: DATABLITZ - REHABILITATION FOR THE REAL WORLD (PART D)
}

\section{Implications for Rehabilitation Drawn From a Correlational Study of Health Related Quality of Life (HRQL) and Neuropsychological Consequences for Children and Adolescents Following Encephalitis}

\author{
E. Talbot ${ }^{1}$, A. Starza-Smith ${ }^{2}$ and A. Hart ${ }^{1}$ \\ ${ }^{1}$ Faculty of Health, Life and Social Sciences, University of Lincoln, Lincoln, United Kingdom \\ ${ }^{2}$ Department of Clinical Psychology and Neuropsychology, Nottingham University Hospitals NHS Trust, \\ Nottingham, United Kingdom
}

$B_{\text {ropsychological, psychological, and medical consequences, making }}^{\text {ackground and aims: Following encephalitis, children can experience neu- }}$ health-related quality of life (HRQL) of particular interest in this clinical population. This study was designed to investigate whether relationships exist between the two most frequently reported neuropsychological consequences of encephalitis (executive function and everyday memory problems) and parent/carer reported HRQL. In addition, it explored to what extent these and other illness specific factors are predictors of parent/carer reported HRQL. Method: This study used an exploratory cross-sectional design. Correlations and multiple linear regression were applied to explore primary and exploratory hypotheses. Thirty-eight parents/carers of children/adolescents, aged 8-15 years old, who had a history of encephalitis responded to an invitation to take part. Participants were recruited through the Encephalitis Society. Each parent/carer completed a Pediatric Quality of Life Inventory ${ }^{\mathrm{TM}}$ (PedsQL $\left.{ }^{\mathrm{TM}}\right)$, a Behaviour Rating Inventory of Executive Function (BRIEF), a Children's Memory Questionnaire (CMQ), and a demographic/illness specific questionnaire in relation to their child. Results: Everyday memory, executive function and sleep difficulties were found to significantly correlate with parent/carer reported HRQL. Combined, these sequelae were found to account for up to $71 \%$ of the variance of parent/carer reported HRQL, with everyday memory being the most significant predictor. Discussion and conclusions: Frequently reported neuropsychological impairments, and sleep difficulties, following childhood encephalitis are found to relate significantly to parent/carer 
reported HRQL. This indicates important implications for children postencephalitis and suggests specific areas for rehabilitation, with a focus on enhancement of executive functions and everyday memory.

\title{
The Use of Alerting Cues in the Rehabilitation of Executive Dysfunction in the 'Real World'
}

L. Birkett-Swan', N. Alderman ${ }^{1}$ and J. Evans ${ }^{2}$

${ }^{1}$ Kemsley, Psychology, St Andrew's Healthcare, Northants, United Kingdom

2 Section of Psychological Medicine, University of Glasgow, Glasgow, United Kingdom

\begin{abstract}
$\boldsymbol{B}^{\text {ackground and aims: Impairment of executive functions is associated }}$ $\boldsymbol{B}_{\text {with functional difficulties because of failure to maintain attentive con- }}$ trol or intention relating to task completion. Nonroutine or novel activities of daily living require an individual to attend to, process and deal effectively with numerous stimuli and competing demands. Manly and colleagues (2004) demonstrated that error rate on such a task carried out in a controlled environment was reduced through presentation of brief auditory cues. The current study will ascertain whether auditory alerting cues are as effective in reducing errors on a non-routine task performed in the context of a 'real world' setting. Method: Twenty participants with acquired brain injury and impairment of executive function undertook a simplified version of the Multiple Errands Test within a shopping centre (MET-SV: Alderman et al., 2003). Participants completed the MET-SV twice in order to accommodate both an experimental and control condition within a counterbalanced design. In the experimental condition they were presented with auditory alerting cues, whilst in the control condition they were not. Results: Data will be presented which demonstrates the impact of alerting cues on MET-SV performance; the hypothesis that cuing exerts a particular influence on rule-breaking errors will be examined. Discussion and conclusions: Presentation of auditory alerting cues whilst individuals with executive impairment undertake nonroutine tasks in natural environments can enhance learning and assist in their rehabilitation. This method may be of special relevance to those who have particular difficulties in maintaining attentive control. Other benefits to rehabilitation, including cost and ease of implementation, will also be discussed.
\end{abstract}

\section{A Case Study on the Effects of Content-Free Cueing and Goal Management Training on Day-to-Day Psychological and Cognitive Functioning}

G. Hardy¹, F. Gracey¹, A. Bateman'1, J. Evans², J. Fish³, E. Greenfield³, J. Ingham', D. Malley ${ }^{1}$, T. Manly ${ }^{3}$ and B. Wilson ${ }^{3}$

' Oliver Zangwill Centre for Neuropsychological Rehabilitation, Princess of Wales Hospital, Ely, United Kingdom

${ }^{2}$ Section of Psychological Medicine, University of Glasgow, Glasgow, United Kingdom

${ }^{3}$ MRC Cognition \& Brain Sciences Unit and the Oliver Zangwill Centre, Cambridge, United Kingdom

Gollowing acquired brain injury (ABI), many people experience problems with planning, organisation, and executing and maintaining behaviours, commonly referred to as 'executive dysfunction'. Such problems can interfere with people's ability to achieve day-to-day goals and have a negative impact upon psychological wellbeing. This case report of TF, a 52-year-old woman with executive dysfunction following $\mathrm{ABI}$, forms part of the Assisted Intention Monitoring (AIM) study, which is a double-blind randomised controlled trial incorporating a placebo-training control condition. The active intervention 
combines brief training in 'goal management' with a subsequent 'contentfree cueing' period, in which participants receive occasional text-message cues that serve to remind them to review their goals, but do not contain any specific information about tasks they have to complete. Over a 12 -week period covering baseline, placebo training and active training phases, TF completed diaries recording achievement of self-identified goals, and undertook a prospective memory task of making phone calls at set points of the day. Assessment of executive functioning and self-reported mood and goal management also took place at 4 points throughout the study. TF's goal attainment and prospective memory performance remained stable over baseline and placebo training phases, and showed significant improvement following goal management training. Furthermore TF reported that she found many of the strategies introduced in the study extremely useful and has continued to use them since completing the study. In conclusion it seems that this approach may be useful in helping people with executive dysfunction to improve goal management and psychological well-being.

\section{Coping With Communication Breakdown Following Severe Traumatic Brain Injury}

J. Douglas and C. Mitchell

Human Communication Sciences, La Trobe University, Victoria, Australia

$\boldsymbol{R}$ ackground and aims: Impaired communication is a well-established con$\boldsymbol{B}_{\text {sequence of traumatic brain injury (TBI). As a result, people with TBI }}$ frequently experience communication breakdown. Typically, we use communication-specific coping strategies in situations characterised by communication breakdown. Productive strategies enhance message transfer and facilitate participation. In contrast, non-productive strategies do little to resolve problems and promote social isolation. The aim of this study was to compare the communication-specific coping strategies of adults with severe TBI and healthy adults. Method: Participants included 26 adults with TBI (posttraumatic amnesia of 7-92 days) and 26 controls matched for gender, age and education. Communication-specific coping was evaluated using the Communication Coping Questionnaire (CCQ). The CCQ has two subscales (Expressive: CCQ-Exp; Receptive: CCQ-Rec), both of which have acceptable internal consistency (CCQ-Exp $\alpha=0.82$; CCQ-Rec $\alpha=0.85$ ) and temporal stability (CCQ-Exp $r=0.80$; CCQ-Rec $r=0.83$ ). Results: Overall, TBI participants reported using significantly fewer communication-specific coping strategies and using them significantly less frequently than matched controls. However, a differential pattern of use was evident across productive and non-productive strategies: participants with TBI used significantly fewer productive strategies and more non-productive strategies than controls. They also used productive strategies less frequently and non-productive strategies more frequently than controls. Discussion: These results indicate the efforts used by people with TBI to cope with communication breakdown are less effective than those of non-brain injured adults. Directly addressing communication-specific coping by reducing the use of non-productive strategies and increasing the use of productive strategies may well improve social interactions. Conclusion: It is important to broaden the focus of intervention beyond communication deficits themselves to the realm of coping with communication breakdown. 


\section{Cognitive Remediation Therapy of Working Memory and Executive Deficits in Asperger Syndrome: Preliminary Findings}

L. Weiner ${ }^{1}$, E. Bizet ${ }^{2}$ and J. Bruckmann ${ }^{1}$

${ }^{1}$ Centre de Ressources Autisme, EPSAN, Brumath, France

${ }^{2}$ Centre de Ressources Autisme, Centre Hospitalier de Rouffach, Colmar, France

$\boldsymbol{B}$ ackground: Asperger Syndrome (AS) is a high prevalence developmen$\boldsymbol{D}_{\text {tal disorder characterised by qualitative impairment in } 3 \text { domains: social }}$ interactions, communication, and behavior (Scott et al., 2002; APA, 2000). Unlike other disorders in the autism spectrum, subjects with AS have average IQ. However, cognitive specificities, such as executive dysfunction and poor working memory abilities have been frequently reported (Williams et al., 2006; Russo et al., 2007). Moreover, these deficits are thought to have an impact on symptoms, quality of life, and social prognosis. Aims: (1) evaluate the effects of cognitive remediation therapy specifically designed to improve working memory (WM) and cognitive flexibility (CF); and, (2) estimate its impact on autism symptoms, psychiatric comorbidities, self-esteem and quality of life. Hypotheses: We expect to reduce the impact of rigidity and poor working memory abilities on the quality of activities, on change management, and on the development of more flexible and reciprocal relationships. These core autism behaviours are thought to be involved in quality of life and the development of psychiatric comorbidities during lifespan (Gaus, 2007). Hypotheses are: (1) significant improvement of WM and CF following specific interventions for each of these abilities; (2) decrease of the intensity of some autism symptoms, such as repetitive behaviour and inflexible social interactions; (3) increase of quality of life and self-esteem measures; (4) correlations between cognitive remediation effects (i.e., WM and CF improvement) and change on the intensity of autism symptomatology, psychiatric comorbidities, self-esteem and quality of life. Methods: 2 participants, aged 18 and 20. ADI-R and DSM-IV-TR diagnostic criteria for AS were fulfilled for both subjects. Questionnaires and comprehensive neuropsychological assessments were administered prior and after cognitive intervention. Numerous specific and nonspecific baselines were performed throughout therapy, in order to measure its effects. 35 individual sessions of 90 minutes were conducted within 5 months: first, 20 aiming to improve WM, then 15 focusing on executive deficits. Sessions took place twice a week. Therapy for WM involved remediation of 3 subcomponents of WM's central administrator: mental effort, updating, and management of interferences (Duval et al., 2007). The program consists of laboratory and ecological pencil and paper exercises hierarchically conceived for each subcomponent. Therapy for executive deficits was inspired by Tchanturia et al. (2004) and Fasotti et al. (2000). It consists of laboratory and ecological cognitive flexibility exercises. Results: Final data collection is ongoing. Therefore, results and discussion will be available in May 2010. 


\title{
SESSION 7: AGING, STROKE, AND DEMENTIA
}

\section{The Influence of Ideomotor and Constructional Limb Apraxia on Disability After the Left Hemisphere Stroke}

\author{
V. Nesterova, V. Grigoryeva and Y. Sagildina \\ Department of Neurology, Neurosurgery and Medical Genetics, Nizhniy Novgorod State Medical \\ Academy, Nizhniy Novgorod, Russia
}

\begin{abstract}
$\mathrm{O}$ ur aim was to determine whether ideomotor (IA) and constructional apraxia (CA) are associated with disability after left hemisphere ischaemic stroke (IS). Methods: 40 patients with apraxia and without significant motor and sensory deficitswere examined after the IS ( $<6$ months). Eight patients (Group 1) exhibited only IA. Thirty-two patients (Group 2) had both IA and CA. The use of real objects, object use pantomime, intransitive movements and hand postures were tested in response to a verbal command and under a condition of imitation to diagnose the form of apraxia. The quantitative assessment of IA was based on the hand postures coping test results (Kimura D., Archibald Y., 1974). CA was quantified by the evaluation of a table and cube drawing and coping task (Glozman J., 1999). Disability was assessed using the Rankin scale (RS). Results: IA severity was not different between the two groups neither in the ipsilateral nor in the contralateral hand. Functional outcome by RS in Group 1 (median [25th, 75th percentile] $0,5[0 ; 1,0])$ was better than in Group $2(2,0[1,0 ; 2,0]), p<$ .05 . There was a moderate correlation between the contralateral IA score and RS in Group 1 and Group $2(-0,42 ;-0,59$, respectively), $p<.05$. The correlation between CA severity and RS in Group 2 was not statistically significant. Conclusions: Results suggest that IA affects everyday activities in stroke patients. The combination of IA and CA has a more negative impact than IA alone.
\end{abstract}

\section{Neurocognitive Aging and Quality of Life in Greek Cypriot Adults}

F. Constantinidou and C. Charilaou

Department of Psychology, University of Cyprus, Nicosia, Cyprus

$O$ bjectives: This study is part of the first systematic research programs exploring neuropsychological-neurocognitive performance and quality of life issues in the elderly Greek-Cypriots. The primary objective was to investigate verbal learning, working memory, executive functioning abilities and quality of life issues in Greek-Cypriots over the age of 60. Participants: Participants were 84 Greek-Cypriot men and women, between 55 to 85 years (mean $=69.4, S D=6.5)$ with average education 9.5 years $(S D=3.9)$ and average MMSE $27.2(S D=1.8)$. One hundred and thirty male/female GreekCypriot adults over 60 from various educational and socioeconomic backgrounds participated in the project. Methods: All participants were screened for global cognitive and memory decline prior to participating in the project. A battery of standardised clinical neuropsychological tests to assess neurocognitive abilities and the WHO Quality of Life (WHOQOL-BREF) questionnaire were administered. Results: Statistical analyses revealed significant relationships $(\mathrm{p}<.01)$ between years of education, quality of life, and cognitive abilities. Specifically, years of education correlated significantly with physical health. In addition, physical health was significantly correlated with psychological health and speed of processing abilities. Both physical and psychological health were correlated with strong naming 
abilities, while psychological health was also positively correlated to social relationships and environmental support. Conclusions: Results indicate that certain aspects of quality of life relate to cognitive performance. In addition, higher education relates to perceptions of physical health in adults over 60 .

\title{
The Impact of a Short-Term Multicomponent Residential Treatment Program on Cognitive Functions and Activities of Daily Living in Persons With Dementia
}

B. Romero', C. Jonas², C. Lahmeyer², F. Müller', M. W. Riepe²

${ }^{1}$ Alzheimer Therapiezentrum Bad Aibling, Bad Aibling, Germany

${ }^{2}$ Department of Psychiatry and Psychotherapy II, Mental Health \& Old Age Psychiatry,

University UIm, Germany

\begin{abstract}
Rackground: To support long-term cognitive functioning and activities of daily living of people with dementia, intervention programs for patients and family caregivers are needed. Aims: To test the hypothesis that a shortterm residential treatment program would improve cognitive functioning and activities of daily living of people with dementia. Method: An ongoing controlled trial with pretreatment and 3-month follow-up recruited 160 people with dementia. The outcome measures are changes in cognitive functions and activities of daily living. The multicomponent program was designed to prepare patients with dementia and caregivers for life with a progressive disease. The program includes: (1) medical treatment and intensive rehabilitation for patients, based on the concept of Self-Maintenance Therapy, and (2) an intervention program for caregivers. Results: Results of the ongoing study will be shown. We predicted that at follow-up that the intervention group will have better outcome relative to the control group on Mini-Mental State Examination (MMST), Alzheimer's Disease Assessment Scale - Cognition (ADAS-Cog) and Bayer-ADL scale (B-ADL). Discussion: The long-term relative stability of cognitive and everyday function can be seen as beneficial for patients.
\end{abstract}

\section{SESSION 8: DATABLITZ - EMOTIONS AND COPING}

\section{Resilience and Executive Dysfunction: A Comparison Between Children With Brain Injury and Noninjured Controls}

J. Tonks ${ }^{1}$, W. H. Williams ${ }^{1}$, I. Frampton ${ }^{2}$, P. Yates ${ }^{3}$ and A. Slater ${ }^{1}$

${ }^{1}$ School of Psychology, University of Exeter, Exeter, United Kingdom

2 Cornwall Partnership NHS Trust, Cornwall, Cornwall, United Kingdom

${ }^{3}$ Royal Devon and Exeter Hospital, Exeter, Exeter, United Kingdom

\begin{abstract}
Children with brain injuries commonly experience difficulties with adaptive behaviours. These difficulties, which are typically linked to executive dysfunction, may be enduring and are associated with particularly poor long-term outcomes. At the individual level, changes in intellectual ability, temperament and communicative skill are commonly problematic, but these changes are embedded in socio-environmental contexts that may serve to protect or reduce a child's sense of resilience. Aims: We wanted to explore: (1) whether there is significant deterioration in a young person's sense of resilience in comparison with peers associated with brain injury; and (2) whether resilience is influential in mediating relationships between executive dysfunction and socioemotional behaviour disturbance after brain injury. Methodology: We administered 'The Resiliency scales for Children and Adolescents' (Prince Embury, 2007), the Beck Youth Index of depression and anxiety and measures of behaviour
\end{abstract}


and executive function with 15 children with brain injury and 70 matched healthy children aged 9 to 15 years. Results: Group differences between the measures will be explored. Conclusions: Resilience may be a useful construct in rehabilitation. Findings will provide indications as to how such factors may be conceptualised within neurorehabilitation.

\title{
Prevalence of Aggression in a Female Brain Injury Unit: Do Women Differ to Men?
}

\author{
L. Birkett-Swan, H. Mortimer and M. Montenegro \\ Kemsley, Psychology, St Andrew's Healthcare, Northants, United Kingdom
}

$\boldsymbol{B}_{\text {common sequelae of brain injury (Tateno et al., 1993; Alderman, 2007). }}^{\text {ackground and aims: It has been well documented that aggression is a }}$ In addition, there is much evidence reported that men without brain injuries are more often aggressive than women and also display more 'dangerous' types of aggression. However, there is very little information about how gender impacts either frequency or severity of aggression or if causes of aggression differ between gender following acquired brain injury (ABI). Method: Aggression was recorded for all service users on female specific brain injury rehabilitation ward over a six month period using the Overt Aggression Scale Modified for Neurorehabilitation (OAS-MNR; Alderman et al., 1997). Collected data included information relating to frequency, type, antecedents and interventions. Results: Despite what is known about differences in gender and aggression in the nonbrain-injured population, aggression in this group of women did not appear to differ significantly to reported studies of aggression in men in similar services. Discussion and conclusion: There are a number of reasons why men and women without brain injuries display different types and severity of aggression. It would appear that within the rehabilitation service examined, some of these are no longer relevant, combined with the loss of inhibitory control observed arising from executive impairment. These changes in both role and biological factors may have a potential implication for the rehabilitation of aggression following ABI, particularly in women.

\section{Increased Self-Efficacy Following Brain Injury Education/Rehabilitation Group Intervention}

A. Champion, N. Jago and A. Thomson

Gloucestershire Brain Injury Team, Gloucestershire Royal Hospital, Gloucestershire, United Kingdom

Rackground and aims: Group interventions are an established element of $\boldsymbol{B}_{\text {brain injury rehabilitation. If one of their aims is for attendees to imple- }}$ ment more strategies more consistently to manage their persisting difficulties, then the concept of self-efficacy is pertinent, as the literature links this with effective self-management of chronic conditions. The aim of the current evaluation was to compare self-efficacy before and after attendance at a sixsession group intervention incorporating information provision regarding the common consequences of brain injury and compensatory strategies for their management. Method: A self-efficacy questionnaire was administered before and after the intervention. The group content included information about the brain and brain injury, fatigue management, goal-setting, a cognitive-behavioural approach to the emotional consequences of brain injury, management of cognitive difficulties including attention, memory and executive function, and input from an expert patient and a return-to-work service. 
Results: Ten attendees with acquired brain injury have completed the outcome measures in this ongoing study. A Mann-Whitney test indicated a statistically significant increase in self-efficacy score following attendance at the group $(p<.05)$. Discussion: If indeed self-efficacy mediates effective implementation of compensatory strategies then the increased self-efficacy seen post-intervention is a positive finding. A number of factors may contribute to this, some of which may relate to the content of the sessions and some to the experience of group attendance. Conclusions: Attendance at a six-week group intervention relating to the management of the consequences of brain injury is associated with increased self-efficacy.

\title{
Does Acute Traumatic Stress Predict Mild Traumatic Brain Injury (MTBI) Symptoms?
}

W.H. Williams ${ }^{1}$, J. Jetten ${ }^{2}$, J. Jones ${ }^{1}$, A. Harris ${ }^{3}$, L. Mounce ${ }^{1}$, A. Reuben ${ }^{3}$ and A. Haslam ${ }^{1}$

${ }^{1}$ School of Psychology, University of Exeter, Exeter, United Kingdom

${ }^{2}$ School of Psychology, University of Queensland, Queensland, Australia

${ }^{3}$ Emergency Dept, Royal Devon and Exeter Hospital, Exeter, United Kingdom

\begin{abstract}
Rackground: Mild traumatic brain injury (mTBI) can lead to physical, emotional, cognitive, and behavioural problems. Clear prognostic indicators are not well established. Recent research has indicated a role for Acute Stress Disorder (ASD) in predicting outcomes post-mTBI. We investigated the recovery process of individuals with mTBI at 2 weeks and 3 months post-injury. Methods: Longitudinal follow up study of mTBI and orthopaedic patients attending an Emergency Department at 2 weeks and at 3 months post-injury. Measures included: Rivermead Post Concussion Scale (RPCS), General Health Questionnaire (GHQ), Trauma Memory Quality (TMQ) and Trauma Screening Questionnaire (TSQ). There were also items regarding pre-injury variables, social network and life satisfaction. Medical records for episode care were checked for trauma details. Results: At Time 1, there were 148 patients (105 with mTBI, of which 44 had "complicated" injury). There were 43 with orthopaedic injury (upper limb). At Time 2, 94 patients were retained. The Complicated mTBI group had higher RPCS scores than the noncomplicated group. Analyses of any differences in trauma injury variables, including memory of event and later reporting of concussion and stress symptoms, will be examined. Conclusions: The consequences of mTBI are difficult to predict. Findings for this study will enable consideration of neurogenic, psychogenic, and social factors as they interact in influencing risk of symptoms over time. Such findings would enable earlier, targeted, rehabilitation for affected individuals.
\end{abstract}

\section{Rediscovering Emotions Again: Systematic Exploration of Self-Reported Absence of Emotion After Probable Ventromedial Frontal Lobe Injury}

S. Palmer and C. Herbert

Kerwin Court, Brain Injury Rehabilitation Trust, West Sussex, United Kingdom

$\boldsymbol{B}$ ackground and aims: Ventromedial frontal lobe injury is associated with Bemotional processing and ability to 'tune in' to one's 'gut feelings' to make decisions, problem-solve and interpret subtle cues about intent in others' communications. We describe the case of a 23-year-old man with acquired difficulties with these tasks, 6 years after severe traumatic brain injury (TBI). He described being unable to feel any emotion. Despite this, he 
demonstrated significant health anxiety and had a post-injury history of aggression within the family setting. Method: We describe a multidisciplinary process that explored biological and psychological explanations for his experience. Based on a biopsychosocial formulation of aggression/lack of emotion, interventions included systematic emotion-monitoring before and after planned behavioural experiments. Heart-rate data confirmed that he experienced physiological arousal in response to psychological threat or agitation. Therefore, heart-rate monitoring, video feedback and Sensecam images were used to support him to 'tune in' to minor emotional fluctuations. Alongside these interventions, psychotherapeutic work focussed around formulation of unconscious internal discrepancy and challenging feared identity that he can no longer 'do the right thing' in social situations and is now fundamentally 'bad' and not worthwhile. Results discussion: We describe a gradual increase in range of emotions reported, improved ability to complete activities of daily living and greater confidence. Self-report was noted to vary between rehabilitation and family contexts. Conclusion: Neuropsychological consequences of ventromedial damage can be exacerbated by physiological, psychological, relational and functional factors. Supporting individuals to address many of these factors can enhance their emotional experience.

\title{
SESSION 9: TECHNOLOGY
}

\section{Can Meditation Help to Improve Control of Brain-Computer Interface (BCI) Devices to Operate Neuroprotheses?}

\author{
A. Jansari ${ }^{1}$ and L. F. Tan ${ }^{2}$ \\ 1 School of Psychology, University of East London, London, United Kingdom \\ 2 Department of Mechatronics and BioMedical Engineering, Universiti Tunku Abdul Rahman, Kuala \\ Lumpur, Malaysia
}

$\boldsymbol{B}_{\text {ture signals from an intact motor cortex can be used to power neuro- }}^{\text {ackground and aims: Brain-Computer Interface (BCI) devices that cap- }}$ prosthetic devices such as artificial limbs or wheelchairs. An important aim in the development of BCIs is the improvement of the signal-to-noise ratio between the wanted motor signal and other background neural signals. Neuroimaging work has shown that various meditation practices can lead to measurable changes in brain activity so the current study was designed to determine whether meditation could improve the efficiency of BCIs. Method: Thirty healthy volunteers were randomly assigned to three groups. A Meditation group studied mindfulness meditation for 12 weeks; a Music control group learned to play the guitar for 12 weeks; while a Control group had no intervention. In addition to the formal lessons, intervention groups had to sustain daily practice. Participants' ability to control a visual cursor simply by imagining moving specified limbs while getting visual feedback was assessed longitudinally at baseline and at a number of points during and after intervention. Results: Compared to the two control groups, participants in the Meditation groups showed the greatest gains in BCI-control efficiency as measured by number of BCI tasks completed and speed of task completion. Discussion and conclusions: The results demonstrate that Meditation training could be used in conjunction with technological developments of neuroprosthetic devices to have significant impacts on the lives of limbimpaired individuals. 


\title{
Internet-Based Cognitive Therapy in Outpatient Settings: An Overview and Personal Experiences
}

\section{A. Barnes}

Practice for Neuropsychological und Psychological Rehabilitation, Bad Ems, Germany

\begin{abstract}
$\mathrm{T}$ The internet has provided new opportunities for offering patients with brain injury cognitive therapy at home following inpatient treatment. This presentation will look in particular at the way in which neuropsychologists working with outpatients can improve their services through internet-based systems. Ideal systems are those which enable the therapist to upload a set of specific exercises for the client onto a system-server, which can then be downloaded by the client at home. The therapist can periodically monitor the client's performance and make appropriate adjustments based on the results. One major advantage of being able to deliver and monitor therapy in the home environment is that the limited time available in therapy sessions, frequently only one hour in a week, can be used more effectively for other important aspects of therapy, for example, treating emotional issues and developing compensatory strategies for everyday activities. Characteristics of currently available tele-therapy for cognitive rehabilitation will be reviewed, together with their advantages and limitations with respect to the needs of clients and therapists in outpatient settings. Personal experiences made with an internet-based system in a neuropsychological practice are described.
\end{abstract}

\section{The Effectiveness of Computer-Assisted Neuropsychological Rehabilitation and the Methods of its Assessment}

A. Bolewska and E. Lojek

Department of Psychology, University of Warsaw, Warsaw, Poland

$\mathrm{T}$ The aim of the study was to examine the effects of computer-aided cognitive rehabilitation in a group of 15 patients with brain damage. The effectiveness of the therapy was measured by improvement on computer tasks, the results of neuropsychological tests, and the quality of life estimation. The subjects suffered from mild to moderate neurocognitive disorders including attention and memory problems as well as aphasia. The experimental procedure involved baseline assessment, 10-week therapy twice a week (20 hours in total) and post-test. Neuropsychological tests for the assessment of attention, memory, language problems and the quality of life were used twice: in the pre- and post-tests. Twelve healthy controls were also examined twice (with a 10-week interval between examinations) using the same battery of neuropsychological tests. The Rehacom program and the Polish computer therapy program for aphasics called Afasystem were used in the rehabilitation. The computer- assisted rehabilitation tasks were selected for each patient individually. The results of the study showed that there was a significant improvement on computer-aided tasks in all subjects with brain damage. However, none or very small improvement was observed on neuropsychological tests when learning effect was controlled. Moreover, only one third of the patients reported a positive influence of the rehabilitation on their quality of life. The results of the study confirm the importance of using different types of measures to estimate the effectiveness of computerassisted neuropsychological rehabilitation as well as the necessity of applying various kinds of therapy to improve cognitive, emotional and social skills of patients with brain damage. 


\title{
SESSION 10: DATABLITZ - SOCIAL FACTORS AND INTERVENTIONS
}

\section{Social Reintegration After Traumatic Brain Injury: The French Experience}

J.-L. Truelle ${ }^{1}, \mathrm{~K}$. von Wild ${ }^{2}$ and M. Montreuil ${ }^{3}$

${ }^{1}$ Neurorehabilitation Department, University Hospital, Garches, France

${ }^{2}$ kvw-neuroscience consulting $\mathrm{GmbH}$, Münster, Germany

${ }^{3}$ Psychology and Psychopathology, University Paris, Paris, France

Traumatic brain injury (TBI) accounts for more years of life lost than breast cancer, stroke and HIV combined. It is a specific handicap, often hidden, mainly involving cognitive and, moreover, behavioural sequelae. Social re-entry is a long-term, fluctuating, and precarious process. The French experience will be illustrated by 4 initiatives addressing 4 challenges specific to TBI:

1. Bridging the gap, between initial rehabilitation and community re-entry, via transitional units dealing with assessment, retraining, social/vocational orientation and follow-up. Today, there are 30 such units based on multidisciplinary teams, the goal of which is mainly implemented by mental rehabilitation. The program lasts for 6 months.

2. Assessing mental recovery by TBI-specific evaluation tools, built throughout an international network: EBIS holistic assessment and, recently, QOLIBRI, a TBI-specific quality of life tool, validated in 6 languages. This subjective outcome measure is mainly based on mental components reflecting specific handicap. It is noteworthy that the prognostic factors of a good community re-entry are almost entirely based on mental recovery: motivation, awareness, emotional control, coping, and ego-identity.

3. Facing medico-legal consequences not sufficiently addressed by mental recovery assessment: In that perspective, we developed guidelines for TBI-specific expert appraisal, including mandatory neuropsychological assessment, family interview and an annual forum gathering lawyers and health professionals.

4. Promoting specific rehabilitation programs founded on limited medication, ecological neuropsychological rehabilitation, exchange groups and workshops, violence prevention, continuity of care, environmental restructuring, family involvement and 'resocialisation'.

\section{Training Paid Caregivers of People With Acquired Brain Injury (ABI): A Qualitative Study}

\author{
N. Behn', L. Togher ${ }^{2}$ and E. Power ${ }^{2}$ \\ 1 Speech Pathology, The University of Sydney, Cambridgeshire, United Kingdom \\ ${ }^{2}$ Speech Pathology, The University of Sydney, Lidcombe NSW Australia
}

Background and Aims: Communication impairments are a common
sequelae for people with an acquired brain injury (ABI) though little
attention is directed towards their everyday communication partners. Paid
caregivers play a vital role in the rehabilitation process; however, they
receive little support and communication skills training. This study will
examine the experience of paid caregivers and evaluate a programdesigned
to improve paid caregivers' conversational interactions with people with an
ABI. Method: 6 paid caregivers participated in a 17-hour communication
training program. It incorporated 'collaborative' and 'elaborative' proce- 
dures (Ylvisaker, 1998) with genre-based activities specific to the caregivers' workplace (Togher et al., 2004). Data was collected from focused interviews before and after the training which was then transcribed and analysed using qualitative thematic analysis to evaluate the outcomes of training. Results: As a result of the training, paid caregivers identified an increased knowledge in how to communicate more effectively with people with an ABI. They expressed improved confidence in using communication strategies and techniques to facilitate conversations. Caregivers also provided information on workplace and training barriers that could be addressed to improve their experience and learning. Discussion and conclusions: This study supports the training and support of paid caregivers for people with an ABI. Improved skills subsequently impacted their ability to fulfil the roles required for supporting a person with ABI. Moreover, the results provide insight to inform rehabilitation professionals and help them identify barriers that will need to be overcome in order to facilitate their interactions with people with ABI.

\section{Clubs in Care: Can These Social Interventions Cure?}

C. Haslam, I. Gleibs and A. Haslam

School of Psychology, University of Exeter, Exeter, United Kingdom

$\boldsymbol{R}$ ackground and aims: There is no doubt that social factors are important in maintaining health, but recommendations suffer from a lack of theoretical specificity. Researchers from the social identity tradition argue that social group membership is critical and suggest that building new social networks can counteract the negative consequences of trauma and illness. We test this view in two studies with older people in care. Method: In Study 1, we randomly allocated residents $(n=46)$ to one of four conditions in which they either participated in activities that focused on consumption of water or on other topics, and were conducted either in a small group or individually, over a period of 8 weeks. Study 2 investigated the impact of gender-based clubs (i.e., Ladies and Gentlemen's Clubs) in residents $(n=26)$ over a period of 12 weeks. Before and after these interventions we measured cognitive ability, social identification, social support, life quality and mood. Results: In both studies we found evidence of improved or maintained well-being (i.e., life quality and mood), but only for group-based interventions. Importantly, there was a clear gender effect in Study 2, with only the Gentlemen showing improvement in mood and social identification. Discussion and conclusions: The findings from these studies provide further support for the importance of social group membership in protecting wellbeing in vulnerable older people and highlight the various forms that interventions can take. We also discuss the implications of the failure to find cognitive improvement, and suggest that such outcomes may require more targeted intervention. 


\title{
Social Cognition, So What? Predictors of Intimacy and Adjustment in Couples' Relationships Following Acquired Brain Injury
}

\author{
G. Yeates ${ }^{1}$, N. Creamer², M. Mahadevan', J. Whitehouse-Hart ${ }^{3}$, F. Gracey ${ }^{4}$, \\ A. Bateman ${ }^{4}$, L. Phillips ${ }^{5}$ and B. Dunn 6 \\ ${ }^{1}$ Community Head Injury Service, Buckinghamshire Hospitals NHS Trust, Aylesbury, Bucks, \\ United Kingdom \\ ${ }^{2}$ Oxford Doctoral Course in Clinical Psychology, University of Oxford, Oxford, United Kingdom \\ ${ }^{3}$ Open University \& Community Head Injury Service, Buckinghamshire Hospitals NHS, Aylesbury, \\ Bucks, United Kingdom \\ ${ }^{4}$ Oliver Zangwill Centre for Neuropsychological Rehabilitation, Cambridgeshire NHS PCT, Ely, \\ United Kingdom \\ ${ }^{5}$ Psychology, University of Aberdeen, Aberdeen, United Kingdom \\ ${ }^{6}$ MRC Cognition \& Brain Sciences Unit and the Oliver Zangwill Centre, Cambridge, United Kingdom
}

\begin{abstract}
Rackground and aims: Compared to many other long-term conditions, $\boldsymbol{B}_{\text {there are unique negative outcomes for couples' relationships following }}$ acquired brain injury. Links have been suggested between the presence of social cognition impairments for the survivor and deteriorating social relationships of all kind. However, little empirical evidence has been produced to support these associations, nor have these been conceptualised within the complexities of psychosocial contexts in which brain injuries occur. Method: 70 Survivors of acquired brain injury (traumatic brain injury, cerebrovascular accident, other-all 18 months or more post-injury) and their long-term romantic partners participated. Survivors completed measures of social cognition (mentalising, social inference, emotional recognition, social judgments, emotion-based decision-making) and other neuropsychological function (memory, attention, executive functioning), Survivors and partners completed psychosocial questionnaires (of anxiety, depression, anger, social support, economic and role arrangements within the family). In addition dependent variables were measures of dyadic adjustment and relationship closeness, completed by survivors and partners. Using this cross-sectional design, a multiple regression analysis was performed on the data. Certain couples also participated in attachment interviews and this qualitative data was triangulated with the statistical analysis. Results: Social cognition factors were found to predict couple relationship outcomes, but only through a mediating influence of certain psychosocial factors. Discussion and conclusions: The results are discussed in relation to socio-emotional attunement processes within the couple relationship, also influenced by other contextual factors (social support, roles). Key implications for couples therapy are considered, notably the prioritisation of affect within therapy sessions.
\end{abstract}

\section{Evidence for a Support Group for People With Multiple Sclerosis}

N. Lincoln, F. Yuill and J. Holmes

Institute of Work, Health \& Organisations, The University of Nottingham, United Kingdom

Introduction: Many people with multiple sclerosis (MS) experience emo-
tional problems (McGuigan and Hutchinson, 2006). Recent guidelines
suggest that patients with chronic illnesses, such as MS, with mild to mod-
erate depression should be treated with psychological therapies (NICE,
2009). The aim was to assess the effectiveness of a group intervention, based
on cognitive behavioural principles, for patients with MS and low mood.
Method: The study was a single blind randomised controlled trial comparing
a psychological group intervention with a waiting list control. Patients with 
MS were asked to complete the Hospital Anxiety and Depression Scale (HADS) and General Heath Questionnaire-12 (GHQ-12). Those who scored $\geq 8$ on either the depression or anxiety subscale of the HADS or $\geq 3$ on the GHQ-12 were recruited. Participants also completed the Beck Depression Inventory, MS Self-efficacy scale, Multiple Sclerosis Impact Scale, EuroQol, Guy's Neurological Disability Scale and a service use questionnaire. Patients were randomly allocated to receive group intervention (six sessions) or usual care, and outcomes were repeated four and eight months after randomisation. Results: Of the 211 patients who were eligible, 151 consented to participate. Currently 113 participants have completed the 4- month follow-up questionnaires, and 77 participants the 8-month follow-up questionnaires. All participants will have completed outcome assessments by June 2010. Groups will be compared. Discussion: If there are significant differences it will suggest that psychological intervention should be provided in clinical practise for those with low mood and that groups are a practical way to deliver such an intervention.

\section{SESSION 11: EMOTIONAL FACTORS}

\section{Defensiveness and Symptom Reporting in the Medico-Legal Context: Empirical Findings and a Case Study}

T. Ownsworth and M. Ono

School of Psychology, Griffith University, Brisbane, Queensland, Australia

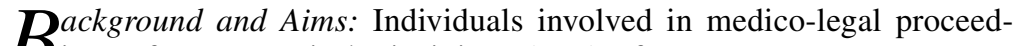
ings after traumatic brain injury (TBI) often report more symptoms than non-claimants. Conversely, higher defensiveness has been found to be associated with lower symptom reporting. The impact of defensiveness on symptom reporting in the medico-legal context has yet to be examined after TBI. Accordingly, this study aimed to investigate the relationship between medico-legal status, defensiveness and symptom reporting. Methods: The sample of 54 individuals with TBI (50\% claimants) were on average 4.3 years post-injury $(S D=2.9)$ and recruited from outpatient or vocational rehabilitation services. Participants completed the Symptom Expectancy Checklist and Marlowe-Crowne Social Desirability Scale (measure of defensiveness) as part of a broader assessment battery. Results: Statistical analysis identified significant associations between level of symptom reporting and defensiveness $(r=-.49, p<.001)$ and medico-legal status $(r=-.30, p<.05)$. Interestingly, claimants displayed significantly lower defensiveness than non-claimants $(p<.05)$. Medicolegal status and defensiveness predicted symptom reporting after controlling for age and severity of TBI $\left(R^{2}\right.$ change $\left.=.23, p<.001\right)$. Further, defensiveness mediated the relationship between medico-legal status and symptom reporting ( $\beta$ reduced from -.30 to -.16 ), with a significant indirect effect observed $(p<.05)$. Conclusions: The findings suggest that individuals' motivation to acknowledge or disclose negative information about themselves may be altered by their experiences in the medico-legal system, which in turn may contribute to higher symptom reporting than nonclaimants. The negative consequences of attempting to maintain a defensive self-presentation style will be briefly illustrated by a treatment case study. 


\title{
A Preliminary Model for Post-TBI Depression
}

\author{
J.F. Malec ${ }^{1}$, A.W. Brown², A.M. Moessner², T. Stump ${ }^{3}$ and P. Monahan ${ }^{3}$ \\ ${ }^{1}$ Rehabilitation Hospital of Indiana and Department of Physical Medicine and Rehabilitation, Indiana \\ University School of Medicine, Indianapolis, United States of America \\ ${ }^{2}$ Mayo Clinic TBI Model System, Physical Medicine and Rehabilitation, Rochester, \\ United States of America \\ ${ }^{3}$ Division of Biostatistics, Indiana University School of Medicine, Indiana, United States of America
}

\begin{abstract}
Rackground and Aims: Depression affects the majority of individuals with

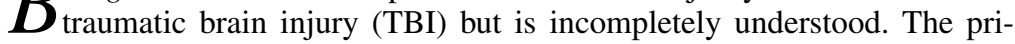
mary aim of this study was to evaluate and further develop a model for postTBI depression. Method: 158 patients admitted to the hospital with moderate-severe TBI were assessed by a TBI Clinical Nurse Specialist using the Mayo-Portland Adaptability Inventory (MPAI-4) Ability Index. Patient self-appraisal of post-TBI ability and depression were reported on the Awareness Questionnaire and Beck Depression Inventory-II, respectively. Outcomes 1-year post-injury were assessed with the MPAI-4 Participation Index. Other variables, i.e., education, TBI severity as indicated by duration of post-traumatic amnesia (PTA), and pre-injury depression, were assessed by interview and review of medical records. The relationships among these variables were examined using Structural Equation Modeling (SEM). Results: Successive SEM resulted in a parsimonious model with excellent fit. Consistent with prior research, a moderately strong association between self-appraisal of post-TBI ability and depression was found. Injury severity was not significantly associated with post-TBI depression. One year outcome was associated with depression and TBI severity. Discussion and conclusions: The strong association between selfappraisal of post-TBI ability and depression is consistent with the cognitivebehavioral model of depression, and recommends consideration and further study of cognitive-behavioral therapy for post-TBI depression. The lack of association between TBI severity and depression may represent the indirect and proxy nature of current measures of TBI severity, e.g., PTA; emerging neuroimaging techniques may provide more direct measures needed to advance this line of research.
\end{abstract}

\section{Factors Associated With Long-Term Post Concussion Symptoms (PCS) Following Mild Traumatic Brain Injury (mTBI)}

N. King ${ }^{1,2}$ and S. Kirwilliam ${ }^{2}$

${ }^{1}$ Community Head Injury Service, The Camborne Centre, Aylesbury, Bucks, United Kingdom

${ }^{2}$ Oxford Doctoral Course in Clinical Psychology, University of Oxford, Warneford Hospital, Oxford, United Kingdom

Rackground and Aims: A small minority of individuals experience long-

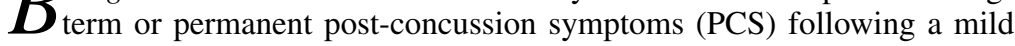
traumatic brain injury (mTBI). There has been no systematic, quantitative research examining a wide range of variables in a representative sample of such patients (i.e., with PCS beyond 1 year post injury). This study explores a broad spectrum of demographic, cognitive, emotional and psychosocial factors (known to be important in the development of early PCS) in a representative sample of patients with long-term PCS. Method: 100 consecutively referred patients to a Community Head Injury Service in Buckinghamshire, UK for the treatment of persistent PCS, at least 18 months post injury were identified and invited to participate. An exploratory design evaluated a range 
of demographic, cognitive, emotional and psychosocial variables and their relationship to PCS severity and quality of life (QOL). Results: 24 participants, with a mean time post-injury of 6.9 years responded. They were characterised by: (1) older age compared to those typically presenting with mTBI; (2) very high levels of PCS; (3) high post-injury unemployment; (4) pre- or postmorbid factors which might exacerbate post concussional difficulties; (5) elevated levels of anxiety and depression; and (6) mildly reduced scores on tests of short-term memory, and speed of information processing. QOL negatively correlated with PCS severity and anxiety scores accounting for $45.9 \%$ of the variance in PCS severity. Discussion and conclusions: Age, pre/post-morbid concomitant factors, neuropsychological deficits and emotional status are key variables in understanding the phenomenon of longterm PCS. Important vulnerability factors in the development of long-term symptoms may therefore be older age and any additional compromise to an individual's emotional or cognitive capacities. Very high levels of PCS, high post-injury unemployment and measurable cognitive deficits can be permanent features of mTBI. QOL is directly related to symptom severity.

\title{
SESSION 12: INNOVATIVE MEASURES
}

\section{Validity of the European Brain Injury Questionnaire (EBIQ) subscales}

\author{
A. Caracuel ${ }^{1}$, A. Bateman ${ }^{2}$ and T. Teasdale ${ }^{3}$ \\ ${ }^{1}$ Clinical Psychology and Neurosciences Institute, University of Granada, Granada, Spain \\ ${ }^{2}$ Clinical management, Oliver Zangwill Centre for Neuropsychological Rehabilitation, \\ Princess of Wales Hospital, Ely, United Kingdom \\ ${ }^{3}$ Psychology, University of Copenhagen, Copenhagen, Denmark
}

Rackground: The European Brain Injury Questionnaire (EBIQ) is an international instrument used for the subjective assessment of the complex disorders after acquired brain injury (ABI). Three very similar subscales (Cognitive, Depression and Impulsivity/Irritability) have been proposed using two different methods: Principal Component Analysis (PCA) and Nonmetric Multidimensional Scaling (NMS). The subscales constructed using those different methods share the names but not exactly the same items. Aim: To apply Rasch analysis for testing the fit to the Rasch model of the subscales built from both methods and to use analysis of Differential Item Functioning (DIF) by country for checking the cross-cultural validity of each subscales. Method: 335 people with ABI from France, Spain and UK completed the EBIQ. RUMM2020 software was used for data analysis. Results: After deleting a very few misfitting items all subscales showed fit to the Rasch model, with a Person Index Separation greater than .80 and unidimensionality. Between the two Cognitive subscales, the best fit to the model was shown for the subscale made using NMS method and it was also free of DIF by country. The best Depression subscale was that from PCA method but both depression subscales shared two items affected for DIF by country. Finally, the best fit and lower DIF by country was shown by the Impulsivity/Irritability subscale based on NMS methodology. Conclusions: The Rasch model can be used to check the psychometric properties of subscales that have been constructed using different procedures and can help decide between two of them. 


\section{The Bangor Gambling Task: A Useful Clinical Tool in the Assessment of Emotion-Based Decision-Making in Survivors of Traumatic Brain Injury?}

A.-L. R. Adlam¹, M. Adams' ${ }^{1}$, R. Rous ${ }^{1}$, G. Yeates ${ }^{2}$, A. Bateman ${ }^{3}$ and F. Gracey ${ }^{3}$

${ }^{1}$ School of Medicine, Health Policy and Practice, University of East Anglia, Norwich, United Kingdom

${ }^{2}$ Community Head Injury Service, Buckinghamshire Hospitals NHS Trust, Aylesbury, Bucks, United Kingdom

${ }^{3}$ Oliver Zangwill Centre for Neuropsychological Rehabilitation, Princess of Wales Hospital, Ely, United Kingdom

Background and Aims: The Bangor Gambling Task (BGT) was designed

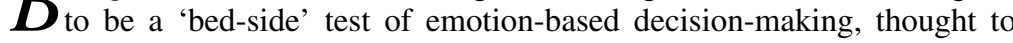
underpin the social and emotional difficulties associated with traumatic brain injury (TBI). However, evaluation of the performance of TBI participants on the BGT has not been carried out. The aims of this study were to: (1) characterise BGT performance in survivors of TBI; (2) investigate the relationship between performance on the BGT and other cognitive measures; and (3) investigate whether BGT performance is related to real-life social and emotional difficulties. Method: Thirty survivors of TBI and 41 age- and education-matched healthy controls completed the BGT and measures of speed of processing, sustained attention, working memory, and executive function. Carers also completed ratings of everyday social and emotional difficulties. Results: Survivors of TBI were impaired relative to controls on the BGT. Discriminant function analysis confirmed that performance on Block 3 effectively classified participants into their respective groups (TBI, control), yielding a cut-off raw score $(-5)$ that can be applied clinically. Performance on the BGT in the TBI group was only associated with speed of processing. Discussion: BGT performance is impaired in survivors of TBI and this is unrelated to executive function and working memory. These data provide a platform for studies examining effects of location and aetiology of brain injury and psychophysiological changes predicted to be associated with BGT performance. Conclusion: The BGT, with its practical advantages over the Iowa Gambling Task, is a useful clinical tool in the assessment of decision-making in survivors of TBI.

\section{Assessment of Prospective Remembering in a Multi-Element Goal Management Task: A Study of the Convergent Validity of the Modified Hotel Test}

S. Baylan and J. Evans

Section of Psychological Medicine, University of Glasgow, Glasgow, United Kingdom

$\boldsymbol{B}_{\text {and }}$ ackound and aims: Everyday life frequently involves multi-tasking prospective remembering. Neurological disease affecting the frontal lobes often disrupts the ability to manage multiple sub-goals/tasks. Clinical assessment tools are therefore required that (1) test the ability to manage multiple goals, (2) identify deficits in specific processes required to manage goals (e.g., prospective remembering), (3) reflect the functions of specific regions of the brain, and (4) predict functioning in everyday life. This study investigated whether performance on a modified version of the multi-element Hotel Test (Manly et al., 2002) was associated with performance on three computerised tests of prospective memory (PM) that have been shown to activate Brodmann's area 10 in the prefrontal cortex (Burgess et al. 2003). Method: Forty healthy adults (mean $=45.4, S D=24.8$, range $=19-78$ ) completed the Modified Hotel Test (mHT) and the three computerised tests of prospective 
memory, each of which has an ongoing task performed alone (OG condition) or with additional prospective remembering demands (PM condition). Results: Performance in the PM condition (collapsed across tasks) predicted the number of prospective memory targets detected on the mHT (rho $=.617$, $p<.01)$. Prospective memory target performance in the PM condition was also associated with the number of tasks attempted on the mHT (rho $=.391$, $p<.05)$. Discussion and conclusion: The study provides evidence for the convergent validity of the mHT task and suggests that the mHT may be a useful measure of prospective memory and goal management in a complex multi-element task environment.

SESSION 13: DATABLITZ - REHABILITATION FOR THE REAL WORLD (PART II) A Single Case Design Involving the Use of an Electronic Assistive Device to Improve Cooking Safety in a Man With Severe Memory Problems

T. Powell and N. Krishnan

School of Psychology, University of Birmingham, Birmingham, United Kingdom

\begin{abstract}
$\boldsymbol{B}$ ackground and aims: We describe an intervention to improve cooking

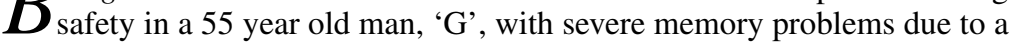
head injury sustained 22 years ago. $\mathrm{G}$ was anxious and avoided cooking because, if he became distracted and moved away from the cooker, he forgot that he was cooking and burned food. This unusual system might be classified as an example of 'orphan technology', such systems have not previously been reported in ABI. Method: An alerting device was developed consisting of a timer located beside the cooker which $\mathrm{G}$ sets and synchronises with an alerting device that he wears. When the cooking period is over, the device tells $\mathrm{G}$ to return to the cooker. After a period of training using an errorless learning approach, $\mathrm{G}$ was able to use the device independently. A daily record of burning food and anxiety about cooking was kept for 4 weeks before training, 2 weeks during training, and 5 weeks after training. Results: Frequency of burning food reduced from $55 \%$ of occasions cooked to zero after implementation. Anxiety about cooking decreased from $9.7 / 10$ prior to training to 2.8/10 after training. Discussion and conclusion: The device soon became part of G's routine and he no longer burns food as the device alerts him to return to the cooker. G says that his self-esteem has improved and he no longer worries about cooking. This study shows that, with appropriate intervention, clinically significant functional improvement is possible even 22 years after injury.
\end{abstract}

\title{
Development of a Patient Focused Organisation for Outpatient Rehabilitation for Persons With Acquired Brain Injury (ABI): Minimising
}

B. Johansson, A. Godbolt, C. Sandström and L. Eriksson

Department of Rehabilitation Medicine, Neuroscience, University Hospital, Uppsala, Sweden

ackground: The nature of impairments related to acquired brain injury
structure; means that these patients are vulnerable to weaknesses in service
services. Additionally, clinical problems are often complex and primary or
acute health-care providers may not have sufficient time or training to direct
a referral correctly. This may lead to serial referrals before an appropriate
service is identified, and there is a real risk that patients' needs are not met. 
We describe development of a multidisciplinary referral management system for patients of working age with acquired brain injury in Uppsala County, Sweden, to address these problems. Methods: A new system for referral evaluation was developed as follows: (1) survey of patients' and relatives' experiences of accessing and receiving rehabilitation services; (2) crossorganisational discussion; (3) establishment of a patient focused referralreview organisation; (4) development of this to an integrated clinical service. Results: A cross-organisational, transdisciplinary referral group was established as a single point for evaluation of all new referrals for outpatient brain injury rehabilitation in Uppsala County. Referrals were accepted from any health care provider or from patients themselves. Of 1291 referrals, 1173 (130/year) were accepted as appropriate. $49.2 \%$ of accepted patients needed only limited services provided by referral group personnel; $66 \%$ of these required only one or two contacts. Discussion and conclusions: Increased focus on patients' needs has minimised unnecessary health care contacts, largely eliminated waiting times, and offered patients an efficient route to individualised rehabilitation service.

\title{
A Cognitive Remediation Program for Everyday Memory Deficits in Children With Acquired Brain Injury
}

\author{
J. Ho', S. Lah', A. Epps ${ }^{2}$, L. Parry² and M. Poole ${ }^{2}$ \\ ${ }^{1}$ School of Psychology, The University of Sydney, Sydney, Australia \\ ${ }^{2}$ Brain Injury Rehabilitation, Sydney Children's Hospital, Randwick, Australia
}

$\boldsymbol{R}$ ackground and Aims: Everyday memory difficulties represent the most

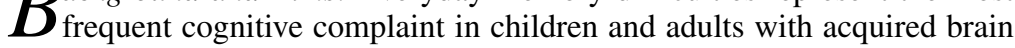
injury (ABI). In adults, the degree of complaint has little or no correlation with results of standard neuropsychological tests. In children, the nature and rehabilitation of everyday memory deficits has not been researched. The aims of this study were to (1) establish neuropsychological correlates of everyday memory deficits in children with ABI, and (2) develop and investigate the efficiency of a cognitive rehabilitation program. Method: Fifteen children and adolescents aged 11-18 who had acquired a significant brain injury over 12 months ago and had difficulties in everyday memory were included in the study. They were seen for pre- and posttraining assessments of everyday memory, attention, declarative memory, executive skills and behaviour. Children attended the memory remediation program consisting of six weekly sessions that involved self-instruction and diary training. Results: Everyday memory difficulties were related to impaired attention and internalising behavioural problems but not to results obtained on standard memory tests. On completion of the rehabilitation program, there was a significant decrease in everyday memory difficulties as reported by parents and children. In addition, children used diaries more frequently. Moreover, significant secondary gains were found in mood (anxiety and depression), but not in cognition. Discussion and conclusions: The results provided preliminary evidence that our 6-week program could be effective in reducing everyday memory difficulties and improving psychological wellbeing in children with ABI. 


\title{
A Comparison of Outcomes Measures for Suitability in Brain Injury Rehabilitation
}

C. Robinson ${ }^{1}$ and J. Leathem ${ }^{2}$

${ }^{1}$ Services for the Elderly, Waikato District Health Board, Tauranga, New Zealand

2 Psychology, Massey University, Wellington, New Zealand

\begin{abstract}
$\boldsymbol{R}$ ackground: Measurement of progress and outcome is essential in brain injury rehabilitation. Of many measures available for this purpose the FIM+FAM is most widely used but is limited in its coverage of the activity limitations and participation restrictions experienced by individuals who have suffered brain injuries. Aims: The current study examined the suitability of six outcome measures (including the FIM+FAM) for use with individual with traumatic brain injury (TBI), in a rehabilitation setting in New Zealand. Method: Six outcome measures (FIM+FAM, BICRO-39, MPAI-4, R-CHART, CIQ, and DRS) were administered to ten participants with brain injuries on admission and after six weeks of rehabilitation, and evaluated on the basis of content (personal care, mobility, physical independence, communication, social interaction/participation in recreational activities, family contact, emotional functioning/adjustment, cognitive functioning, productive activity, financial independence, transportation) and agency factors (absence of ceiling/floor effects, quick/simple to understand/score/use, meaningful concluding interpretations, information collected identifying specific individual/family rehabilitation goals specific, space for input from the client and family, measures change over time). Results: Results suggested that no one outcome measure adequately indicated levels of activity limitations and participation restrictions for people with TBI in the agency. Although the FIM+FAM was found to contain a number of strengths particularly in assessing physical independence, the MPAI-4 was more useful in identifying goals related to activity limitations and participation restrictions. Discussion: Possible approaches to combining subscales for these measures are discussed.
\end{abstract}

\section{Participate! Evaluation of Participation Specific Effects in German Outpatient Neurorehabilitation}

D. Poeppl ${ }^{1}$, W. Fries ${ }^{2}$, R. Deck ${ }^{3}$, C. Pott ${ }^{4}$, S. Fischer ${ }^{5}$, G. Risse ${ }^{6}$ and P. Reuther ${ }^{2}$

${ }^{1}$ ANR Ahrweiler - Center for Outpatient Neurorehabilitation, Ahrweiler, Germany

${ }^{2}$ BV ANR - German Federal Association of Outpatient Neurorehabilitation, Munich, Germany

${ }^{3}$ Research Departement, Institute for Social Medicine Luebeck, Luebeck, Germany

${ }^{4}$ Physiotherapy, Center for Outpatient Neurorehabilitation and Neuropsychology Prof. Fries, Munich, Germany

${ }^{5}$ Neuropsychology, Center for Outpatient Neurorehabilitation and Neuropsychology Prof. Fries, Munich, Germany

${ }^{6}$ ANR Ahrweiler - Center for Outpatient Neurorehabilitation, Ahrweiler, Germany

Rackground and aims: The International Classification of Functioning, $\boldsymbol{D}_{\text {Disability and Health (ICF) is anchored in the German social legisla- }}$ tion, which states 'participation and self-determined conduct of life' as the ultimate goal of rehabilitation of social insurance. To date a lack of conceptual clarity in defining and explaining participation hindered the development of commonly accepted and methodologically appropriate tools to measure participation. In addition, in German neurorehabilition, there is a lack of use of already existing instruments to measure 'participation'. Therefore, we conducted a multi-center study in 6 German outpatient neurorehabilitation centers evaluating participation and assumed moderator 
variables. The data was compared with results of a sample of inpatients in an unpublished study. Method: Status of participation was assessed at admission and discharge in the course of rehabilitation and at 4-month follow-up with the Mayo-Portland Adaptability Inventory (MPAI) and a German questionnaire (Index zur Messung von Einschraenkungen der Teilhabe, IMET). Additionally, clinical experts were asked to rate patients' participation status at admission and discharge. Data were analyzed with common descriptive and inferential statistics. Results: Preliminary results show small to medium effects of an increasing level of participation in stroke outpatients at discharge and follow-up. Patients with degenerative neurological diseases could maintain their level of participation. None of these effects could be found in the inpatient sample. Discussion and Conclusions: For a broad part of neurological patients with different patterns of diseases, outpatient neurorehabilitation showed moderate effects on patients' participation 4 months after discharge. Inpatient rehabilitation seemed to achieve more effects on functional abilities.

\title{
POSTERS - DAY 1
}

\section{An Inpatient Survey of Poststroke Sleep Problems}

\author{
S. Anderson, G. Dampney and S. Griffith \\ Sussex Rehabilitation Centre, South Downs Health Trust, West Sussex, United Kingdom
}

Background and aims: Sleep disturbance is common following brain $\boldsymbol{D}_{\text {injury and has the potential to disrupt rehabilitation efforts through its }}$ effects on fatigue levels and daytime alertness (Oellet \& Morin, 2006). The important role of sleep in the rehabilitation of stroke patients has become increasingly recognised (Sterr et al., 2008). To further explore this area, we conducted a questionnaire-based survey of sleep quality in 25 rehabilitation inpatients post-stroke. Our aims were to assess the nature and extent of sleep difficulties, to identify those most likely to experience disturbance, and to explore contributing factors. Methods: A questionnaire designed to investigate sleep quality, latency, duration, efficiency and daytime sleepiness was devised and administered to 25 stroke patients. Participant demographics were: 14 males/11 females; average age of 75; average time since stroke of 40 days. Study participants also received a cognitive (Addenbrooke's Cognitive Examination-Revised; ACE-R) and emotional screen (Hospital Anxiety and Depression Scale; HADS). Results: Data analysis revealed that just over half of participants felt that sleep quality had been adversely affected by their stroke, with the majority citing restless sleep as the primary difficulty. Noise was the most frequently reported environmental factor and poor sleep hygiene was regularly evident. Self-reported anxiety showed the most significant correlation with overall sleep quality. Discussion and conclusions: The study findings provide insightful data on the nature and extent of post-stroke sleep difficulties and suggest possible outcomes ranging from the routine provision of ear plugs to behavioural strategies encouraging better sleep hygiene. Exploration of the potential influences of medication and poststroke sleep apnea would be useful study extensions. 


\title{
The Assessment of Interpersonal Skills in Stroke Patients
}

M. Turner and D. Andrewes

Psychology, University of Melbourne, Victoria, Australia

\begin{abstract}
$\boldsymbol{B}$ ackground: Impaired interpersonal communication skills and related depression are common after a stroke necessitating the development of therapy programs to improve emotional processing. Suitable measures of emotional processing to use in a therapy program for depressed stroke patients, however, are not available. Method: Both a verbal and a nonverbal version of an emotion-in-faces task were developed to assess static emotion recognition and two versions of a videoed emotional-scenario task alternating between a male and a female actor were developed to assess dynamic emotional recognition, understanding and response. These tasks were then used to investigate the state of emotional processing in 25 patients with stroke and 31 control patients with rheumatic/orthopaedic disorders. Participants were randomly assigned to either the verbal or the nonverbal emotion-in-faces task and one of the versions of the emotional-scenario task. Related cognitive and emotional processing deficits were also assessed to determine their influence on emotional processing. Results: Right hemisphere brain damage was found to significantly impair emotional perception and emotional understanding, and depression was found to significantly impair the ability to respond to an emotional situation in an appropriate manner. There was no relationship between emotional processing and deficits in cognitive and emotional resources. However, a lack of insight from right hemisphere brain damage may interfere with the results. Conclusion: These findings demonstrate that both tasks may be suitable measures of emotional processing in patients with stroke and indeed in all patients with brain damage. Findings could successfully be incorporated into psychotherapy for poststroke depression.
\end{abstract}

\section{Verbal Fluency Improves Significantly After Cognitive Remediation in First Episode Psychosis}

\author{
and M. Gutierrez ${ }^{3}$ \\ ${ }^{1}$ Psicología y Educación, Universidad de Deusto, Bilbao, Spain \\ 2 Servicio de Psiquiatría, Hospital de Cruces, Barakaldo, Spain \\ ${ }^{3}$ Servicio de Psquiatría, Hospital Santiago de Vitoria, Spain
}

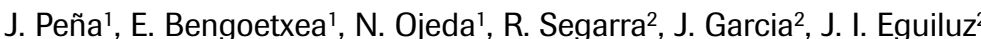

Background: Verbal fluency deficits have been pointed out as a possible
endophenotype in schizophrenia (Szöke et al., 2008). However, whether
these deficits are specific or linked to general semantic-verbal deficits remains
unclear. Additionally, this cognitive domain is already affected in early psy-
chosis and does not improve despite early clinical interventions. Objective:
Authors tested the efficiency of a cognitive intervention specifically developed
for improving fluency in psychosis. Material and Methods: Ninety patients
with first-episode psychosis were randomly assigned to one of two groups:
Cognitive rehabilitation group (REHACOP) or occupational therapy. Patients
in the REHACOP group received 1 month of structured group rehabilitation
sessions ( 3 per week) to improve fluency. Repeated assessments of semantic
fluency and phonological fluency were conducted before and after the treat-
ment. Results: Compared to occupational therapy, the experimental group pro-
duced significant additional improvements in phonological fluency $(F=6.87$,
$p<.01)$, but not in semantic fluency $(F=0.61$, NS). The composite verbal
fluency score was also significantly improved $(F=4.65, p<.05)$. The 
improvement remained 3 months after the treatment end. Conclusions: Cognitive treatment using REHACOP has proven to be effective in treating phonological fluency deficits in first-episode psychosis, whereas socialisation or communication in group therapy by itself is not. The differential pattern shown by semantic fluency is consistent with the proposal of Szöke et al. 2008, who suggest that semantic fluency is a putative endophenotype for schizophrenia with links to a genetic basis compared to phonological fluency.

\section{Predicting Parenting Stress in Caregivers of a Child With a Brain Tumour}

E. Bennett ${ }^{1}$, M. English ${ }^{2}$, A. Starza-Smith ${ }^{3}$ and M. Rennoldson' ${ }^{1}$

${ }^{1}$ Institute of Work, Health and Organisations, University of Nottingham, Nottingham, United Kingdom

2 Oncology Department, Birmingham Children's Hospital NHS Trust, Birmingham, United Kingdom

${ }^{3}$ Clinical Psychology and Neuropsychology, University of Nottingham, Nottingham, United Kingdom

Rackground: Parents of children diagnosed with brain tumours are likely to $B_{\text {face many ongoing stressors and challenges. However, parenting stress has }}$ been little investigated in this population. The current study aimed to identify potential factors that may contribute to parenting stress in these caregivers. Method: The study was cross-sectional and recruited participants from a clinical database at a specialist children's hospital. Parents of children diagnosed with a brain tumour were sent questionnaires, which measured factors related to stress. Stress levels were measured using the Parenting Stress Index Short Form (PSI/SF). Correlation analysis and multiple linear regression were used to examine associations between parenting stress and coping styles, locus of control, child disability, and time since diagnosis. Results: Thirty-seven parents participated and, of these, $51 \%$ were experiencing clinically significant levels of stress. The mean stress level of parents in the study was significantly higher than PSI/SF norms $(t=4.70, p<.001)$. Regression analysis revealed that external locus of control and coping by accepting responsibility accounted for $65 \%$ of the variance in parenting stress. Other styles of coping, child behaviour problems and time since diagnosis were not predictive of stress levels. Discussion and conclusions: There was a high prevalence of parenting stress in caregivers of children with a brain tumour. An external locus of control and coping by accepting responsibility increase the likelihood of elevated levels of stress. Results emphasised the importance of ongoing support for parents. Intervention might helpfully be centered on strategies to increase parents' internal locus of control.

\section{Managing an 'Unmanageable’ Patient Following Two Traumatic Brain Injuries}

T. Bhugobaun ${ }^{1}$ and G. Florschutz ${ }^{2}$

' SCU, Raphael Medical Centre, Tonbridge, Kent, United Kingdom

${ }_{2}^{2}$ RMC, Raphael Medical Centre, Tonbridge, Kent, United Kingdom

Rackground: We report the case of PM, a 42 year old man who sustained $\boldsymbol{D}_{\text {traumatic brain injuries (TBIs) including frontal lobe damage, following }}$ two road traffic accidents when he was 16 years and 24 years of age. Aim: To demonstrate improvement following a structured program designed to reduce spitting, sexually inappropriate behaviour, physical aggression, destructive behaviour, obsessive compulsive disorders (OCDs) and psychiatric treatment. Methods: There were several strands to the treatment program. The main elements were: (1) PM was admitted to a safe, secure therapeutic milieu; (2) his previous medication was reviewed and changed; (3) a 2-week baseline study documented the extent of his problems; (4) the problems were treated with a 
combination of medication, behavioural treatment, psychotherapy, and integrative treatment modalities. Results: PM's documentation and staff reports showed that his inappropriate behaviour, destructive behaviour and mental health needs were significantly reduced. Discussion: Although PM still shows some difficult, challenging or inappropriate behaviour, such as, obsessive compulsive disorders, most of his antisocial behaviours have reduced. In addition family members and staff report that it is much easier to communicate with him and engage in social activities. Conclusion: Despite two serious TBIs and 25 years of being moved from one institution to another, it is possible to reduce socially inappropriate and self destructive behaviour through a therapeutic milieu, medication and rehabilitation program.

\title{
The Use of Feedback for Time Perception Deficit Compensation: A Single-Subject Experimental Design
}

\author{
P. Covre', C. Ford ${ }^{2}$, O. F. A. Bueno', A. Bateman² \\ ${ }^{1}$ Psychobiology, Universidade Federal de Sao Paulo, Sao Paulo, Brazil \\ 2 Clinical Psychologist, Oliver Zangwill Centre, Ely, United Kingdom
}

\begin{abstract}
A lthough altered time perception (TP) is a relatively common complaint after brain injury, it is not usually assessed and investigated. To our knowledge, there is no literature on interventions to improve TP in this population. We aimed to examine the efficacy of feedback during TP tasks. An A-B-A treatment-withdrawal single-subject design was used. A 45-year-old male, previously fit and educated to $\mathrm{PhD}$, suffered bilateral dissection of the carotid artery with near occlusion following a fall, resulting in hemiparesis and multiple cognitive impairments. He was asked to estimate or produce intervals of different durations $(5-15 ; 25-35 ; 55-65$ seconds). During the treatment phase (B) feedback was given regarding the accuracy of his responses. Time estimation for daily activities was also assessed; in this more functional task, the subject timed himself performing activities for feedback. Before intervention, he consistently underestimated the passage of time (formal task rate $=$ .53 ; real task rate $=.50$ ) and overproduced intervals (rate $=3.43$ ). During treatment, he was able to adjust his responses as feedback was given (estimation = .92 ; production $=1.86$ ), but this effect did not generalise from session to session and did not endure after treatment withdrawal (estimation $=.50$; production $=2.15$ ). He reported finding the feedback for the daily tasks helpful, even though he could not perfectly re-estimate the time for all the tasks (mean rate = 1.30). Conclusions: Giving feedback on TP tasks does not remediate the deficit but can help with its compensation by giving an immediate reference for comparison on formal TP tasks, and, most importantly, a reference for better time management for daily activities.
\end{abstract}

\section{Measuring Behavioural Outcome in Neurodisability: Use of the Overt Aggression Scale-Modified for Neurorehabiltation (OAS-MNR) as an Indicator of Service Efficacy}

\author{
N. Alderman 1 , C. Knight ${ }^{2}$, I. Stewart ${ }^{2}$ and A. Boothby ${ }^{3}$ \\ ${ }^{1}$ National Centre for Brain Injury Rehabilitation, St Andrew's Healthcare, Northampton, United Kingdom \\ ${ }^{2}$ Older People and Huntington's Disease, St Andrew's Healthcare, Northampton, United Kingdom \\ ${ }^{3}$ Operational Director, St Andrew's Healthcare, Northampton, United Kingdom
}

$\boldsymbol{B}$ ackground and aims: While determining the efficacy of specialist rehabilitation for nonhomogenous clinical populations is most valid when 
undertaken individually, indicators that provide 'snapshots' that illustrate the general effectiveness of services to commissioners and other stakeholders is essential. Incorporating data from a routinely employed observational rating scale of aggression within the outcome 'basket' may be appealing, especially with regard to savings in time spent training staff, and the reliability and validity of the data obtained. Method: The Overt Aggression Scale - Modified for Neurorehabilitation (OAS-MNR) captures important information regarding aggressive incidents. Data recorded in a 3-month period for 79 patients was used to create an aggregate aggression score (AAS) for each patient taking into account severity of aggression. Results: AAS is a responsive measure that is more sensitive to change in aggressive behaviour than frequency. Using each service user as their own control and calculating the percentage difference between their most recent AAS score and that for the first 12 weeks was shown to be an outcome indicator increasing the sensitivity to behaviour change from that of using the difference between median values alone by between $10.4 \%$ and 42.1\%. Discussion and conclusions: OAS-MNR provides a valuable 'snapshot' that can help inform commissioners and other stakeholders about how effective a service is. Comparison of median ASS scores across time makes for the best visual 'snapshot' of efficacy, while an improvement indicator for a service calculated by determining individual change made since admission provides the optimum solution.

\section{Personal Navigation and Narrative Approach: Benefits and Limitations of Their Incorporation into Brain Injury Rehabilitation}

W. Kühne and P. Frommelt

Neurological Rehabilitation, Asklepios Clinic Schaufling, Schaufling, Germany

The aim of a more profound understanding of the processes contributing

to our outcome data after brain injury rehabilitation resulted in two approaches: the adaptation of Sternberg's concept of personal navigation and the incorporation of narrative approaches. Adding information from qualitative narrative interviews to results of neuropsychological assessment and questionnaires supports the understanding and explanation of successes and failures in long-term outcome. We report data from an evaluation of 54 clients we interviewed an average of more than 5 years after their head trauma: $57 \%$ of the clients were able to work productively; $68 \%$ were living independently or with a partner; $31 \%$ with their parents. None was institutionalised. From the assessment data we extracted necessary abilities and context factors for social and vocational integration. For 5 cases, we will present additional information based on qualitative interviews both with the clients with brain injury and their significant others. For these cases, we will illustrate the specific benefits and limitations of incorporating and operationalising the concept of personal navigation into rehabilitation and evaluation after brain injury. 


\title{
Social Cognition Rehabilitation in Traumatic Brain Injury (TBI) and Posttraumatic Stress disorder (PTSD): Emotion Perception and Expression Interventions
}

K. McCoy ${ }^{1}$, C. Campbell ${ }^{2}$ and M. Kelly ${ }^{2}$

${ }^{1}$ War-Related Injury \& IIIness Study Center, Veterans Affairs Medical Center, Washington, United States of America

2 Polytrauma Program, Veterans Affairs Medical Center, Washington, United States of America

\begin{abstract}
Rackground: Psychosocial functioning plays a significant role in both readjustment after traumatic brain injury (TBI) and in recovery from posttraumatic stress disorder (PTSD). Effective social interaction relies on the successful application of numerous cognitive functions that are prone to disruption by TBI and PTSD, including: emotion perception in self and others, social problem-solving, implementation of intentions, evaluation of outcomes and motivation. There are no known treatments designed for individuals with both TBI and PTSD, and existing treatments do not address the complex factors involved in successful social reintegration. Methods: Clinicians at the Washington, DC Veterans Affairs Medical Center have developed a prototype social cognition group for veterans with co-morbid TBI and PTSD that is anchored in existing research and firsthand clinical knowledge. The group is divided into three interrelated modules aimed to: (1) improve emotion perception in self and others, (2) enhance social problem-solving, and (3) encourage identity development and social readjustment. Results: The Emotion Perception and Expression module includes interventions targeted to improve: static and dynamic affect recognition in others, attentiveness to one's internal feelings, reading and conveying body language, awareness of one's emotional displays ('social self-awareness'), emotional mimicry, sarcasm detection, theory of mind, distress tolerance, matching content to tone of voice, humour, self-disclosure, and gathering additional social information. Initial clinical outcomes are described through illustrative real-life vignettes. Discussion: For individuals with overlapping cognitive and emotional symptoms, an integrated neuropsychotherapy approach holds promise. Implications and plans for further research are discussed.
\end{abstract}

\section{Social Cognition Rehabilitation in Traumatic Brain Injury (TBI) and Posttraumatic Stress Disorder (PTSD): Social Problem-Solving Interventions}

M. Kelly ${ }^{1}, \mathrm{~K} . \mathrm{McCoy}^{2}$ and C. Campbell ${ }^{1}$

1 Polytrauma/Psychology, Veterans Affairs Medical Center, Washington, United States of America

2 War-Related Injury \& IIIness Study Center, Veterans Affairs Medical Center, Washington, United States of America

Rackground: Psychosocial functioning plays a significant role in both

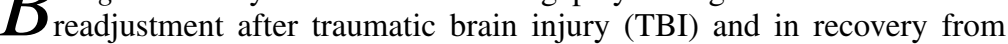
posttraumatic stress disorder (PTSD). Effective social interaction relies on the successful application of numerous cognitive functions that are prone to disruption by TBI and PTSD, including: emotion perception in self and others, social problem-solving, implementation of intentions, evaluation of outcomes and motivation. There are no known treatments designed for individuals with both TBI and PTSD, and existing treatments do not address the complex factors involved in successful social reintegration. Methods: Clinicians at the Washington, DC Veterans Affairs Medical Center have developed a prototype social cognition group for veterans with comorbid TBI and PTSD that is anchored in existing research and firsthand clinical knowledge. The group is divided into three interrelated modules aimed to: 
(1) improve emotion perception in self and others, (2) enhance social problem-solving, and (3) encourage identity development and social readjustment. Results: The Social Problem-Solving module includes interventions targeted to improve: giving and taking in conversations, self-calming, understanding the social context, diffusing conflicts, examination of social schemas, avoiding misunderstandings, assertiveness, topic maintenance, empathy and active listening, asking for and accepting help, explaining your injuries to others, word-finding anxiety, dealing with unexpected outcomes, and managing misperceptions about TBI and PTSD. Initial clinical outcomes are described through illustrative real-life vignettes. Discussion: For individuals with overlapping cognitive and emotional symptoms, an integrated neuropsychotherapy approach holds promise. Implications and plans for further research are discussed.

\section{Social Cognition Rehabilitation in Traumatic Brain Injury (TBI) and Posttraumatic Stress Disorder (PTSD): Identity and Readjustment Interventions}

C. Campbell ${ }^{1}$, M. Kelly ${ }^{1}$ and K. McCoy ${ }^{2}$

1 Polytrauma Program, Veterans Affairs Medical Center, Washington, United States of America

2 War-Related Injury \& IIIness Study Center, Veterans Affairs Medical Center, Washington, United States of America

Rackground: Psychosocial functioning plays a significant role in both $\boldsymbol{B}_{\text {readjustment after traumatic brain injury (TBI) and in recovery from }}$ posttraumatic stress disorder (PTSD). Effective social interaction relies on the successful application of numerous cognitive functions that are prone to disruption by TBI and PTSD, including: emotion perception in self and others, social problem-solving, implementation of intentions, evaluation of outcomes and motivation. There are no known treatments designed for individuals with both TBI and PTSD despite this common comorbidity, and existing treatments do not address the complex factors involved in successful social reintegration. Methods: Clinicians at the Washington, DC Veterans Affairs Medical Center have developed a prototype social cognition group for veterans with co-morbid TBI and PTSD that is anchored in existing research and firsthand clinical knowledge. The group is divided into three interrelated modules aimed to: (1) improve emotion perception in self and others, (2) enhance social problem-solving, and (3) encourage identity development and social readjustment. Results: The Identity and Readjustment module includes interventions targeted to address: understanding your injury, changing roles following injury, how to convey respect, effective versus offensive behaviors, rediscovering role functioning, social anxiety, ability and disability, advocating for yourself, significant other relationships, new psychosocial goals, identifying social norms, establishing safety, circles of care, parenting, and significant other relationships. Initial clinical outcomes are described through illustrative real-life vignettes. Discussion: For individuals with overlapping cognitive and emotional symptoms, an integrated neuropsychotherapy approach holds promise. Implications and plans for further research are discussed. 


\title{
Family Expectations About Recovery After Acquired Brain Injury
}

G. Riley ${ }^{1}$, G. Bellamy ${ }^{1}$ and A. Brennan ${ }^{2}$

${ }^{1}$ Psychology, University of Birmingham, Birmingham, United Kingdom

2 Inpatient neurorehabilitation, Birmingham, United Kingdom

\begin{abstract}
$\boldsymbol{B}$ ackground and aims: The expectations of family carers about recovery after brain injury merit investigation. They may influence the carers' emotional status, and the extent to which they engage in the rehabilitation process. They may also create conflict between the carer and rehabilitation professionals. This study investigated the development of these expectations in the acute and early post-acute stages. Method: This was a qualitative study, using Interpretative Phenomenological Analysis (IPA). Participants were relatives of people with an acquired brain injury who were in-patients on a post-acute specialist rehabilitation unit. Results: Staff management of expectations played a key role. In the acute phase, participants had been led to expect the worst. When this did not occur, there was a loss of trust in staff predictions. In the post-acute phase, staff were less willing to make predictions. However, this did not stop participants from thinking about future progress. Instead of being guided by staff opinions, they resorted to making use of less reliable sources as the basis for their predictions (e.g., their observations of other patients). Information-processing biases were evident in the way in which they dealt with these sources of information (e.g., ignoring or minimising evidence that was contrary to their prediction). At the post-acute stage, most, but not all, participants were optimistic about the future. Optimism was associated with a better overall mood and greater enthusiasm for getting involved in the rehabilitation process. Discussion and conclusions: The implications of the study for how staff manage family expectations will be discussed.
\end{abstract}

\section{Memory Control Beliefs, Strategy Use, and Cognitive Failures in Midlife: The Moderating Effects of Selection, Optimisation, and Compensation}

G. Scheibner and J. Leathem

School of Psychology, Massey University, Wellington, New Zealand

$\boldsymbol{B}$ ackground: Research shows a high prevalence of subjective forgetful-

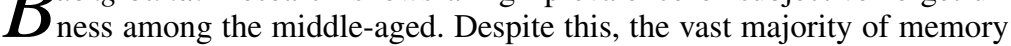
research focuses on the elderly with moderate to severe memory impairment. As a result, very little is known about the nature of forgetfulness that $60 \%$ of middle-aged adults are worried about and perceive as an impediment. Using the theory of selective optimisation with compensation (SOC), this research examines age-appropriate forgetfulness among healthy, middle-aged New Zealanders. Method: An internet-based survey method was used to examine the moderating effects of SOC on memory control beliefs, memory strategy use, and everyday forgetfulness. Results: Regression analyses indicated that SOC accounts for a significant amount of memory control beliefs but not strategy use or cognitive failures. The remaining hypotheses which predicted that SOC endorsement would prove to be beneficial in the contexts of commitment to high performance levels in everyday memory tasks and experiences of age-related memory decrements were not supported by the current findings. Discussion: The findings are considered in the context of methodological limitations: (1) general limitations of self-report assessment; (2) the SOC measure was domain general rather than memory specific; and (3) low internal reliability for some of the SOC subscales. Besides methodological 
limitations, it is argued that individuals in midlife may primarily rely on proactive coping (PC) rather than reactive coping (i.e., SOC). Conceptually, $\mathrm{PC}$ is equivalent to the SOC model and because of the importance of PCrelated behaviours to the aging process it is suggested that $\mathrm{PC}$ would provide a valuable addition to the existing SOC model.

\title{
Strategy Training to Improve Outcomes in Stroke Rehabilitation: A Case Study
}

E. Skidmore', M. Holm¹, E. Whyte ${ }^{2}$, D. Dawson ${ }^{3}$ and J. Becker ${ }^{2}$

${ }^{1}$ Occupational Therapy, University of Pittsburgh, Pittsburgh, United States of America

${ }^{2}$ Psychiatry, University of Pittsburgh School of Medicine, Pittsburgh, United States of America

${ }^{3}$ Department of Occupational Science \& Occupational Therapy, University of Toronto, Toronto, Canada

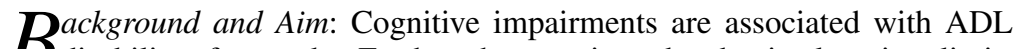
disability after stroke. Further, they can impede adaptive learning, limiting optimal engagement in and benefit from the rehabilitation process. Selfmonitoring strategy training is a promising intervention that may address these problems. The aim of this study is to describe our experiences administering a self-monitoring strategy training program (Cognitive Orientation to daily Occupational Performance) during inpatient rehabilitation after acute stroke. Method: The participant was a 31-year-old male 8 days after ischemic stroke. He sustained mild impairment in attention and executive functions and severe impairment in delayed memory and visuospatial functions (Repeatable Battery of Neuropsychological Status, Delis-Kaplan Executive Functioning System). He participated in daily 45-minute strategy training sessions during inpatient rehabilitation. We examined changes in rehabilitation engagement scores (Pittsburgh Rehabilitation Participation Scale, 0-6), and ADL scores (Functional Independence Measure, 18-126). Results: The participant demonstrated the ability to learn and apply the strategy to address 3 goals, developing plans, and monitoring his own progress during inpatient rehabilitation. All 3 goals were met before discharge. He continued to use the strategy to address 5 additional goals by week 12 . Goals addressed personal care, instrumental and work-related ADLs. Rehabilitation engagement improved from 3 to 5 during his 14 day inpatient rehabilitation stay. ADL scores improved from 68 (moderate disability) at admission, to 97 (minimal disability) at discharge and 126 (independent with basic ADLs) at week 12. Discussion and conclusions: Self-monitoring strategy training during inpatient rehabilitation was feasible with this participant.

\section{Neuropsychological Long-Term Sequelae After Hemorrhagic Isolated Cerebellar Stroke During Development}

\author{
K. Wingeier ${ }^{1}$, S. Bigi ${ }^{1}$, M. El-Koussy², T. Heinks-Maldonado' ${ }^{1}$, E. Boltshauser ${ }^{3}$ \\ and M. Steinlin ${ }^{1}$ \\ ${ }^{\prime}$ Pediatric Neurology, University Children's Hospital, Inselspital Postal, Bern, Switzerland \\ ${ }_{2}$ Diagnostic and Interventional Neuroradiology, University Hospital, Inselspital, Bern, Switzerland \\ ${ }^{3}$ Pediatric Neurology, University Children's Hospital, Inselspital Postal, Zürich, Switzerland
}

ackground: The extent of influence of the cerebellum on developing
cognitive functions is unknown. However, there are signs that it might
play a crucial role. Consequently, the topic of cognitive disturbances after
cerebellar lesions during development has become of increasing interest.
The aim of the present study was to assess long-term sequelae after cerebel-
lar lesions during childhood and adolescence. Method: Eight participants
with isolated focal hemorrhagic cerebellar stroke before the age of 16 were 
recruited. All patients underwent a neurologic exam including the Zurich Neuromotor Assessment (ZNA), the International Cooperative Ataxia Rating Scale (ICARS) as well as an extensive neuropsychological battery assessing a broad range of cognitive functions. Quality of life and possible behaviour/emotional problems were assessed with different questionnaires. Results: The results revealed adaptive fine motor problems (ZNA) and restricted oculomotor movements (ICARS). Conversely, no overall neuropsychological pattern could be identified except marginally reduced reaction times and susceptibility to interference. Furthermore, borderline results in semantic and phonological word fluency tasks were apparent. However, verbal performance and reading abilities were non-pathologic. The majority of the participants reported good quality of life without major physical restrictions or emotional disturbances. Discussion: In this patient group, the presence of a cerebellar cognitive affective syndrome as frequently described in the literature cannot be confirmed. Nevertheless, individual neuropsychological impairments are present. Moreover, fine motor problems were common. Probably heterogeneity of age at stroke and exact lesion site (as well as its afferent and efferent connections to the cerebrum) may have lead to interpersonal differences in neuropsychological outcome.

\section{Elucidating the Psychosocial Context of Post-Concussion Syndrome (PCS): A Case Study From Family Therapy}

G. Yeates ${ }^{1}$ and M. Luckie $^{2}$

${ }^{1}$ Community Head Injury Service, Buckinghamshire Hospitals NHS Trust, Aylesbury, Bucks, United Kingdom

${ }^{2}$ Clinical Psychology for Older Adults, North Essex Partnerships Foundation NHS Trust, North Essex, United Kingdom

Rackground and aims: The literature on post-concussion syndrome (PCS) B following mild head injury includes biopsychosocial formulations. However, it is contended that the complexities of the psychosocial remain conceptually underdeveloped, the family context in particular. Method: The importance of psychosocial factors is illustrated via a case study from family therapy. The work described is with grandparents, a mother and daughter. The latter two both initially experienced PCS following the same road traffic accident, yet demonstrated contrasting outcomes in the year post-injury. The authors worked with the family on the psychosocial level, focusing on communication and provision of material and psychological support within the family. Results: The different outcomes for the two women were formulated to arise from the following social contextual influences: discontinuities in identity situated within family communication and interactional patterns, family life-cycle transitions, together with unequal distribution of material power bases and processes. In working with these factors, the therapy team was able to support mother and daughter to achieve comparable post-injury individual and family goals. Discussion and conclusions: A subgroup of those experiencing enduring PCS has long been identified in the literature and these clients present themselves frequently in brain injury and mental health services. However little has been published on both critical psychosocial predictors of outcome following PCS, or ideas for provision of family support for this group. This case study adds to a small literature to suggest family therapy as a credible and useful component of service provision. 


\title{
Complex Rehabilitation Care at the Military Rehabilitation Centre Slapy and Vltavou (Prague-West)
}

\author{
P. Kulistak and E. Bolcekova
}

Psychology, Military Rehabilitation Centre Slapy nad VItavou, Slapy nad VItavou, Czech Republic

$\mathrm{T}$ The aim of this report is to inform our colleagues about the situation in neuropsychological care of patients with brain damage in the Czech Republic, particularly at the Military Rehabilitation Centre Slapy nad Vltavou (MRC). MRC treats patients after serious neurological, orthopaedic and internal diseases, total endoprothesis and injuries. The therapeutic team consists of physicians and other specialists: physiotherapists, ergotherapists, aphasiologist, nutrition therapist, as well as a neuropsychologist and a neuropsychotherapist. The latter two attend mainly to clients with cerebrovascular accidents, traumatic brain injury, brain neoplasms, and other brain damage. Their goals include cognitive rehabilitation, and reducing emotional and personality problems of clients. They also work with clients' motivation to ensure their cooperation in all areas of rehabilitation. Treatment procedures optimised for this specific group of clients rise from the teamwork of the aphasiologist, ergotherapists, neuropsychologist and neuropsychotherapist. They create individualised neuropsychological programs (computer-assisted cognitive training, neurofeedback) and psychotherapeutic intervention (individual and group therapy) for each client. Another important contribution of MRC is integration of patients with brain damage into a wide community where there are also people without such issues. Unfortunately, there is almost no follow-up care after releasing clients from MRC - this is a general problem of rehabilitation care in the Czech Republic. There are a few specialists (mostly clinical psychologists) who attempt to continue therapy after the client is dismissed; however, in most cases the work is limited to cognitive rehabilitation, and other aspects of therapy are neglected.

\section{Psychological Intervention With a Child Experiencing Reflex Anoxic Seizures: A Case Report}

\author{
E. Bennett ${ }^{1}$ and E. Meldrum ${ }^{2}$ \\ 1 Institute of Work, Health and Organisations, University of Nottingham, Nottingham, United Kingdom \\ ${ }^{2}$ CAMHS, Nottinghamshire Healthcare Trust, Nottingham, United Kingdom
}

$\boldsymbol{B}^{\text {ackground and aims: We describe the case of a ten-year old girl who }}$ B experienced anoxic seizures in response to medical instruments and settings. The girl was referred to psychology and received around fifteen weekly sessions of therapy. We summarise the key strategies and principles of the intervention and outline the positive impact of the work on both the girl and her family. Methods: Intervention utilised an integrated approach and involved psychoeducation about anxiety, graded exposure to feared stimuli (e.g., blood pressure monitors), family work, and the use of narrative therapy techniques. It particularly focused on enhancing the girl's ability to cope with triggers and the reduction of unhelpful avoidance behaviours. Results: By the end of the initial treatment phase, the girl was able to have her blood pressure taken by a nurse, tolerate medical settings and watch procedures on the television. Despite increased contact with triggers, she had 
not experienced any seizures since starting therapy. Both the girl and her parents reported large reductions in anxiety about seizures and increases in their belief in her ability to cope. Standardised measures reflected a reduction in the girl's anxiety and depression levels, and in her mother's parenting stress levels. Discussion and conclusions: In this case, psychological intervention offered effective support in the management of anoxic seizures. Techniques can be used to reduce the frequency of seizures, to enhance self-efficacy and to lower family anxiety levels. Medical professionals should consider referring children experiencing reflex anoxic seizures and their parents for psychological intervention and support.

\title{
A Metacognitive Aapproach for the Treatment of Anxiety and Excessive Ruminations Following Acquired Brain Injury
}

\author{
L. Birkett-Swan and M. Montenegro \\ Kemsley Unit, Psychology Department, St Andrew's Healthcare, Northants, United Kingdom
}

\begin{abstract}
$\boldsymbol{R}$ ackground and aims: Alongside other conditions, such as depression, anxiety is particularly prevalent in people with acquired brain injury (ABI). Wells (1990), on the basis of the Metacognitive Theory of Psychological Disorders, suggested that a style of thinking called the Cognitive Attentional Syndrome is responsible for psychological disorders, including anxiety. Attention Training Technique (ATT), is one of the Metacognitive Approaches that has been evidenced to produce positive results for specific anxiety disorders. Following an ABI, already compromised executive functioning is further confounded by attentional resources being occupied by perseverative ruminations. Given the overlapping concepts observed in both the underlying model of ATT and the supervisory attention system of executive functioning, theoretically it makes sense that this approach would work well with an individual with executive impairment and anxiety typified by ruminative styles of thinking. Method: A single case is presented of a young man who experienced significant and persistent ruminative thoughts that were causing a barrier to him participating in neuro-rehabilitation. Results: The outcome and application of ATT is illustrated through the use of the case in which the individual who had been unable to engage in other forms of psychological interventions experienced improvement in both styles of thinking and frequency of ruminative thoughts. Discussion and conclusion: The process of the intervention will be discussed along with issues that were encountered using ATT with an individual with ABI including challenges experienced. This case builds on the limited evidence to demonstrate the efficacy of Metacognitive Approaches with individuals with ABI.
\end{abstract}

\section{The Cornerstone of Neurorehabilitation: Two Cases of Interdisciplinary Assessment With Contrasting Outcomes}

N. Goudie ${ }^{1}$, D. Kersel ${ }^{1}$ and S. Petrie ${ }^{2}$

${ }^{1}$ Clinical Neuropsychology, Community Treatment Centre for Brain Injury, Glasgow, Scotland

2 Occupational Therapist, Community Treatment Centre for Brain Injury, Glasgow, Scotland

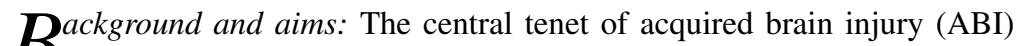
rehabilitation is that of the interdisciplinary team model with an emphasis on functional outcome related to increased participation (ICF: WHO, 2001). While much has been written about the importance of collaboration between disciplines in achieving this model, there are fewer published exam- 
ples of interdisciplinary working in practice. The aim of the current presentation is to provide evidence of the collaborative approach in assessment of two clients with ABI, with varying complex needs. Method: Two contrasting cases will be described to illustrate the approach taken between Clinical Neuropsychology and Occupational Therapy within a community-based neurorehabilitation service. AB (age 39) and CD (age 52) were referred by Social Work for assessment of rehabilitation needs and appropriateness of their current environment. Standardised and non-standardised assessments were administered in addition to interviews with the client, family members and support staff. Results: Assessment of the two clients provided distinct outcomes with the need for one client to move from the community to supported accommodation and the other to move from residential care to the community. Recommendations were also provided for ongoing rehabilitation for the clients and training for family and support staff. Discussion/Conclusions: The two case studies demonstrate the practice of collaborative work between Clinical Neuropsychology and Occupational Therapy. Discussion will highlight the similarities between the two disciplines, their unique, specialist skills and the way in which individualised functional outcomes can be achieved with this approach.

\title{
Mnemonic Enhancement of Cognitive Behavioural Therapy: A Single Case Study and Conceptual Considerations for Therapy Adaptation After Brain Injury
}

\author{
F. Gracey ${ }^{1}$, R. Brindley ${ }^{2}$ and A. Bateman ${ }^{1}$ \\ ${ }^{1}$ Oliver Zangwill Centre, Cambridgeshire Community Services, Ely, United Kingdom \\ 2 Doctoral program in clinical psychology, University of Hertfordshire, Hatfield, United Kingdom
}

Rackground: The emotional consequences of acquired brain injury are

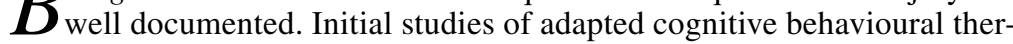
apy (CBT) are promising. However, there has been no systematic study of specific adaptations to CBT to address specific impairments or therapy processes. We present the findings of: (1) a single case experiment, and (2) a single case clinical outcome study with our client Allan who had an acquired brain injury resulting in autobiographical memory and executive impairments and debilitating anxiety symptoms. Method: In study 1, we compared strategies to support recall of thoughts and feelings relating to an anxiety-triggering event, including reviewing images of the event taken with a wearable digital camera ('SenseCam'). In study 2 , we evaluated an adapted CBT protocol that included the review of images taken with SenseCam to support recall and learning. Results: Study 1 demonstrated improved recollection of both event details and of thoughts and feelings for review of SenseCam images compared with a standard therapy diary and no strategy. Study 2 showed the clinical intervention was effective in terms of goal attainment, reduced anxiety symptoms and belief ratings of key cognitions associated with Allan's anxiety. Discussion and conclusion: While there are methodological limitations to both studies, we conclude the findings support the potential use of SenseCam as a memory aid. Furthermore the findings highlight the potential specific capacity for this device to enhance recall of personally salient information about thoughts and feelings within CBT. Implications for the conceptualisation of CBT adaptations after brain injury are considered. 


\title{
Self-Appraisal of Executive Dysfunction in Poststroke Patients
}

\section{Grigoryeva, V. Nesterova, E. Gusanova and Y. Sagildina}

Department of Neurology, Neurosurgery and Medical Genetics, Nizhniy Novgorod State Medical Academy, Nizhniy Novgorod, Russia

\begin{abstract}
$A$ ims: To assess the self-appraisal of executive dysfunction in post-stroke patients and to evaluate whether it correlates with emotional state. Methods: 48 post ischemic stroke patients ( $\leq 6$ months) and 25 age and gender matched healthy control subjects were evaluated by means of detailed neuropsychological assessment, Hospital Anxiety and Depression scale and Russian version of the Dysexecutive Questionnaire (DEX; 'BADS Russian Translation (C) 2009 by Pearson Assessment. All rights reserved'). The total sum of differences between the patients' and observers' scores on items which were rated higher by the patient than by the observer (S1) and total sum of differences between the patients' and observers' scores on items which were rated lower by the patient (S2) were calculated for the DEX analysis. Results: Compared to controls, patients had significantly higher S1 and anxiety and depression levels. Average S1 and S2 revealed no significant differences in the right and left hemisphere stroke patients. Patients' performance was significantly worse on attention, verbal short memory, verbal fluency, psychomotor speed, concept formation and planning, although no patients had dementia. Elevated S1 was associated $(p<.05)$ with deteriorated nonverbal planning (maze tracing test), verbal fluency deficit (phonemic fluency test) and increased anxiety and depression. Conclusions: Most of the patients tend to overestimate their executive dysfunction in the first half year after stroke. Such overestimation combines with non-dementia cognitive decline and increased anxiety/depression levels. Future studies may help to reveal if such overestimation has an influence on the efficacy of patients' rehabilitation post-stroke.
\end{abstract}

\section{To Tell or Not to Tell - That is our Question: An Exploration of Self-Disclosure After Brain Injury}

B. Hagger and G. Riley

School of Psychology, University of Birmingham, Birmingham, United Kingdom

Dreliminary findings of a three-stage study looking at individuals and family carers whose lives have been affected by traumatic brain injury (TBI) will be presented. We asked about their willingness/unwillingness to disclose information to others regarding the effects of the injury. Examples of disclosure and concealment will be provided, as well as the reasons behind those decisions. Information about the study and the findings are presented in a question and answer format. Preliminary results indicate that disclosure and concealment are used as forms of impression management used within the family in an attempt to protect each other and outside the family as protection against perceived and actual stigma and discrimination. The next two stages, the development and validation of a questionnaire, will focus on those who are recovering from a TBI. It is hoped these findings will help us to understand how concealment and disclosure affects self-esteem and the avoidance of social situations. These factors are important as they play a major part in successful rehabilitation and returning to a social life after TBI. 


\title{
Games-Evaluation Task (GET): The Development of a Computer-Based Multi-Tasking Game
}

\author{
S. Hynes, T. Manly and J. Fish \\ MRC Cognition and Brain Sciences Unit, Cambridge, United Kingdom
}

This poster reports the findings of two related studies evaluating the use
of a new computer-based multiple errands-type task, the GamesEvaluation Task (GET). Study 1 investigated the influence of instruction manipulation on GET performance while study 2 focused more on the associations between the task and other established measures of cognitive functioning. A total of 31 adults between 55 and 70 years of age (20, study $1 ; 11$, study 2) were assessed on the GET. Results from study 1 found no difference in participant performance in the three experimental groups when participants were given irrelevant instructions as well as relevant instructions or just the instructions relevant to successful completion of the task. Study 2 confirmed relationships that were shown in study 1 where older adults who reported more frequent everyday cognitive lapses performed less well on the GET, and significant associations were seen between lower IQ and worse performance on the GET. Careful interpretation of the results is necessary at this developmental stage of the GET with the small group that was involved in the study but results justify further testing of the psychometric properties of the measure.

\section{Clinics for Children With Mild-Moderate Acquired Brain Injury (ABI) at a Centre for Rehabilitation}

\author{
C. Jacobsson, I. Sjöberg and M. Kihlgren \\ BarnReHab Skåne, Lund, Sweden
}

\begin{abstract}
Child and Youth Rehabilitation Centre in Skåne (BarnReHab Skåne) is a centre for children (age 0-20 years) at all levels of brain injury. Children with mild-moderate head injury seldom receive any further care from the hospitals/health centres after the immediate care. The effects of the head injury are usually observed in daily life or in school as learning disabilities, behaviour disturbances and difficulties within social interaction. These symptoms can be difficult to interpret as a result from the head injury and are often misunderstood. At Child and Youth Rehabilitation Centre in Skåne (BarnReHab Skåne), we have established a clinic with paediatric neurologist and neuropsychologist where we receive referrals for children with mildmoderate head injury. The aim of this study was to describe our work with rehabilitation activities for children referred to our clinic. We also aim to investigate how parents and children have benefited from the consultation. To describe the group, information extracted from medical journals in our database and background data (cause of the injury, primary symptoms and rehabilitation activities, and so on) were collected. A questionnaire regarding occurrence of different symptoms (e.g., headache, fatigue) and how they experienced the consultation were sent out to all parent/children visiting our clinic during 2006-2009. Data from the questionnaire and the description of the patient group will be presented as well as examples of different rehabilitation implements.
\end{abstract}




\title{
Acalculia Rehabilitation Treatment
}

M. Khrakovskaya

Institute of the Human Brain, Russian Academy of Sciences, Saint-Petersburg, Russia

\begin{abstract}
Rackground: Based on the psychic functions' systemic structure theory and on the neuropsychological analysis of brain lesion-caused deficiencies structure (Luria, 1962), one can make distinction between primary acalculia resulting from derangement in the spatial base of the numeral system (following parieto-occipital brain lesion), and secondary calculation-skill disorders following aphasia/visual agnosia. Primary acalculia involves misperception of numbers' content and position within the numeral system. Method: The proposed method reflects the idea that rehabilitation treatment may be optimised by application techniques based on the processes involved in normal cognition and the system's rules which are automated in healthy adults. School teaching of the four fundamental operations ensures understanding of the first-decile numbers' content, the addition-and-subtraction rules reaching over to another decile, multidigit-number manipulations, and tabular calculation skills explained in an explicit, visual manner observing strict spatial correlations between components. When these skills are consolidated, calculations come to be largely based on speech. Language expresses the numeric-symbol system by numerals ordered in fixed consecutive series reflecting the numeric system's spatial structure through speech stereotypes. The proposed calculation-ability rehabilitation method includes a special system of strictly ordered exercises using different speech (= numeric) sequences viewed as a 'rigid' context regenerating connections between numbers in both their image and verbal aspects. Manipulations with numerical series enable gradual transition to calculation operations. Results: This method has proved effective in primary acalculia cases and for aphasiacaused calculation-ability disorders. Discussion: The experience of neuropsychological rehabilitation in Russia puts recovery of disturbed functions among the priority areas of therapy.
\end{abstract}

\section{The Experience of Care Staff Delivering Reminiscence Sessions to People With Dementia}

\author{
S. Pullan ${ }^{1}$ and C. Knight ${ }^{2}$ \\ ${ }^{1}$ School of Psychology, University of Leicester, Leicester, United Kingdom \\ 2 Older People and Huntington's Disease, St Andrew's Healthcare, Northampton, United Kingdom
}

Rackground and aims: Despite cognitive deterioration associated with dementia, research suggests people can maintain 'personhood' with a supportive surrounding social psychology (Kitwood, 1997). Reminiscence is a popular intervention for people with dementia which potentially improves relationships with care staff. The experiences of care staff delivering reminiscence sessions to people with dementia have not been thoroughly explored. Method: Eleven participants delivered life storybook sessions to individuals with dementia. They were interviewed and transcripts analysed using IPA to explore their experiences Results: Six superordinate themes emerged: barriers to personhood; meeting the person through life storybooks; rehumanising the person; rehumanising the professional; a changed relationship; and plans for the future. Discussion and conclusions: Involving care staff in delivery of life storybook sessions to individuals with dementia in their care is an intervention which could potentially effect powerful systemic change. Individual differences in reactions to reminiscence and the 
content of life storybooks should be considered by the clinical team. In a task focused environment, adding life storybook sessions to the timetable can help to remind staff to monitor the psychological needs of people in their care. Care staff working in this way may experience powerful emotions in themselves and the person they are working with, and feel ill equipped to deal with these. Support and supervision should be offered to help staff develop these skills.

\title{
Inappropriate Sexual Behaviour and Dementia: An Exploration of Staff Experiences
}

L. Hayward ${ }^{1}$ and C. Knight ${ }^{2}$

'School of Psychology, University of Leicester, Leicester, United Kingdom

${ }^{2}$ Older People and Huntington's Disease, St Andrew's Healthcare, Northampton, United Kingdom

Rackground and aims: Research assessing the impact of Inappropriate ${ }_{\text {Sexual Behaviour (ISB) on staff working in dementia care is limited and }}$ there is insufficient awareness of the clinical impact on staff and clients. Studies from forensic and brain injury settings indicate that staff regard ISB as uniquely challenging requiring them to acknowledge and manage their personal and cultural values. Developing an understanding of ISB may have positive implications for staff (e.g., training, supervision) in addition to potentially enhancing the quality of care both for people with a diagnosis of a dementia and their families. Method: Interviews with fourteen staff working within an inpatient setting were undertaken and analysed using a social constructivist Grounded Theory methodology. Results: The core category, 'A Question of Attribution' was identified, describing the process experienced by staff when confronted with ISB. Four main categories were inducted representing participant's initial experiences, their contextualising of behaviour, their process of interpretation and methods of management. Discussion and conclusions: The findings from this study provide a valuable account of the complex psychological and social processes associated with staff experiences of ISB exhibited by older people with a dementia and will help to enrich a growing body of literature regarding ISB exhibited by persons with neurological impairment (Guay, 2008; Johnson et al., 2006; Knight et al., 2008). The impact of ISB should be more routinely considered in preparing staff for working with dementia, and adequate support structures for staff through supervision, peer support and more ad hoc provision should be ensured.

\section{Methods of Neuropsychological Rehabilitation of Patients With Amnestic Syndrome}

\author{
I. Kulikova ${ }^{1}$, N. Varako ${ }^{1}$ and V. Daminov ${ }^{2}$ \\ ${ }^{1}$ Neurorehabilitation, National Medical-Surgery Centre, Moscow, Russia \\ ${ }^{2}$ Neurorehabilitation Department, National Medical-Surgery Pirogov Centre, Nizhnaya, Russia
}

ackground and aims: It is known, that amnestic syndrome is very dis-
abling and resistant to correction. We have been trying to define the most
effective combination of methods for neuropsychological rehabilitation of
patients with amnestic syndrome as result of organic brain damage.
Methodology: the system of methods included: (1) diagnostics - investigat-
ing the neuropsychological syndrome (Luria Battery), paying special atten-
tion to executive functions and individual memory characteristics; (2)
memory training - training in different modalities, with various types of
memory tasks (e.g., 'mechanical' memorising training - trying to memorise 
material of different volume with different time intervals between presentation and reproduction; advancing from easy to more complicated tasks; using personal associations as the basis for memorising; revealing the structure, using it as the basis); (3) memory defect compensatory measures making up schedules of everyday activities and fixing the events, and forming a habit of constant self-inspection; using algorithms - for example, (a) what I remember and not, (b) what I need to remember, (c) how I can do it; using 'technical' tools - for example, organisers, mobile phones, tape recorders, and so on; (4) psychological adaptation. We always started with orientation and forming an adequate idea of the current situation. The explanatory and psychotherapeutic work was provided, where possible. Results and Discussion: Using this system of methods in most cases had a positive influence on memory and orientation. Most helpful was often the 'association' method and effectively tailored compensatory measures. The most effective method was always the result of combining various methods and practicing an individual approach.

\section{Transcranial Direct Current Stimulation: A New Strategy to Improve Effects of Neurorehabilitative Treatments}

\section{P. Polanowska}

2nd Department of Neurology, Institute of Psychiatry and Neurology, Warsaw, Poland

Transcranial direct current stimulation (tDCS) is a well-tolerated tech1 nique of painless and noninvasive brain stimulation that modulates human cortical excitability. This method aims to polarise brain tissues due to flow of weak constant electric current (1-2 mA) between two surface electrodes placed on the scalp. Depending on the polarity, strength, and duration of the stimulation, tDCS can increase (anodal tDCS) or depress (cathodal tDCS) excitability within the stimulated region lasting minutes to over one hour after current offset. It has been documented that t-DCS-induced modifications of brain activity are associated with motor, sensory, and cognitive alterations. It makes the method useful not only for exploring cortical functions in healthy subjects but also for facilitating treatments of various neurological disorders. Clinical consequences of brain injuries are not simply the direct result of an initial insult, but also effects of dynamic activity changes in disrupted neural networks, some of which might be maladaptive and impair recovery. Exposure to direct current polarisation may help in specific and selective enhancement of adaptive patterns of activity, suppression of maladaptive activation patterns, and restoration of activity across bihemispheric neural networks. Indeed, recent small studies of DC stimulation in stroke patients demonstrated that excitability-enhancing anodal tDCS of specialised cerebral cortices improves motor and cognitive functions and modulates perception. Maximum behavioural gains are found when stimulation is coupled with specific behavioural training. High hopes are associated with tDCS-assisted therapy for TBI. There have been also reports about the positive impact of tDCS in neurodegenerative diseases. 


\title{
Cognitive Measures Versus Social Behaviour Measures to Demonstrate the Efficiency of Programs of Neuropsychology Rehabilitation in Chronic Patients
}

\author{
O. García Sánchez and C. González Agudo \\ Neuropsychology, Polibea, Center of Rehabilitation of Acquired Brain Injury, Madrid, Spain
}

\begin{abstract}
$\boldsymbol{B}_{\text {ackground: Neurorehabilitation is required in the acute, post-acute and }}$ $\boldsymbol{D}_{\text {chronic phases after brain injury. Although there is scope for improve- }}$ ment in functioning associated with rehabilitation in the chronic phase, in Spain, there is inconsistency with regard to service provision, emphasis of approach to intervention and measurement of outcome across the different phases post-injury. The present study is concerned with the question of what are the best measures of outcome of neurorehabilitation interventions applied in the chronic phase after brain injury. In particular we compared the impact of holistic neuropsychological rehabilitation interventions on measures of cognition and of social behaviour. Method: Six subjects with acquired brain injury (an average of 4 years post injury) participated in an interdisciplinary rehabilitation program. Social behaviour assessed with Frontal Systems Behaviour Scale (FrSBE) and measures of cognitive functions (attention, memory, language and executive functions and speed of processing) were administered before and after the program. Results: The social behaviour measures were more sensitive to change following the rehabilitation program than cognitive measures and appear to be better measures of the efficacy of the programs of rehabilitation in chronic patients. Discussion: Measurement of outcomes from rehabilitation interventions for patients in the chronic phase after brain injury should focus on measures of behaviour and functioning rather than measures of cognition.
\end{abstract}

\section{Influences of Cognitive Impairment on Motor Recovery}

\author{
E. Skidmore1, M. Holm¹, E. Whyte ${ }^{2}$ and J. Becker ${ }^{2}$ \\ ${ }^{1}$ Occupational Therapy, University of Pittsburgh, Pittsburgh, United States of America \\ 2 Psychiatry, University of Pittsburgh School of Medicine, Pittsburgh, United States of America
}

Rackground and aim: Individuals frequently sustain motor and cognitive $\boldsymbol{B}_{\text {impairments after stroke. This pilot study examined whether individuals }}$ with cognitive impairments were as likely to benefit from an intensive motor rehabilitation program as individuals without cognitive impairments. Method: We recruited participants with mild to moderate unilateral hemiparesis due to stroke who were referred for an outpatient occupational therapy motor rehabilitation program. Benefit from the program was defined as a minimally clinical important difference in Action Research Arm Test (ARAT) total scores 4 and 24 weeks after program onset. Cognitive impairment at program onset was defined as greater than 1 standard deviation below age-adjusted norms on the Repeatable Battery of Neuropsychological Status Total Index score. Results: We enrolled 16 participants, 10 with cognitive impairments. Although not statistically significant, individuals with cognitive impairments demonstrated lower baseline ARAT scores (27.30 vs. 36.17). Nine of 16 participants demonstrated benefit from the motor rehabilitation program at week 4 , and 9 of 11 participants demonstrated benefit at week 24. Among those who benefited at both time points, 6 of the 9 were individuals with cognitive impairments. Relative risk estimates suggest that individuals with cognitive impairments were 1.25 times more likely than participants without cognitive impairments to benefit at week 4, and 3.70 times more likely to benefit at week 24. Discussion and conclusions: 
Preliminary findings indicate that participants with cognitive impairments in this small sample did benefit from the intensive motor rehabilitation program. Findings may be attributed to greater baseline motor impairment, thus greater opportunity for improvement.

\title{
A Single Case Study on the Time Course and Long-Term Efficacy of Alertness Training
}

\author{
W. Sturm and J. Hauke \\ Neurological Clinic, Section Clinical Neuropsychology, University Hospital RWTH University Aachen, \\ Aachen, Germany
}

\begin{abstract}
$\boldsymbol{B}$ ackground and aims: Little is known about time course and long-term $\boldsymbol{B}_{\text {efficacy of training-induced improvement. The present study evaluates }}$ the time course of attentional advancement by repeatedly testing in-between individual training sessions. The outpatient trained had severe alertness deficits following brainstem encephalitis in 2003 and since then achieved no full recovery. Method: Ms. P participated in 15 alertness treatment sessions (45 minutes each of Cogniplus computerised attention training) on 15 separate working days over a 3-week period. Attention functions were assessed by neuropsychological tests 4 years, 1 year, and immediately before the therapy, after every third training session, and three times after the termination of therapy. Furthermore, a self-report questionnaire measured subjective experiences. In order to compare the performance between training sessions, critical differences based on normative data and reliabilities were used to analyze the data. Results: After only three consecutive training sessions, Ms. $\mathrm{P}$ already showed significant improvement of alertness up to the normal range. Furthermore, after 2 weeks, she felt more energetic and more able to concentrate. Even 6 months after the end of the training, the improvement remained stable. Discussion and conclusion: Accelerated training-induced recovery as well as the long-term effects of the training seem to depend on intact cortical structures. In this way, the top-down alertness system could be re-activated by the training procedure in order to insert top-down control on the impaired brain stem arousal structures.
\end{abstract}

\section{Gender Differences in Self-Image After Intensive Neurorehabilitation}

\author{
M. Vink ${ }^{1}$, E. Scherder ${ }^{2}$, M. Holleman ${ }^{1}$, M. Luijpen ${ }^{1}$, A. Beerlage ${ }^{1}$ and R. Horst ${ }^{1}$ \\ ${ }^{1}$ Rehabilitation Center Amsterdam, Amsterdam, The Netherlands \\ 2 Vrije Universiteit Amsterdam, Amsterdam, The Netherlands
}

ackground and aims: Studies on the role of gender in the rehabilitation
outcome of people with traumatic brain injury (TBI) are limited and
findings of the various studies appear to be inconsistent. Little is known
about the relationship between gender and changes in self-image after TBI.
Besides the role of sex differences in the brain and sex hormones in outcome
after TBI, sex differences in cognitive functioning and adjustment to brain
injury have been postulated. As insight into the self-image of women and
men with TBI could be an important determinant for the effectiveness of a
rehabilitation program, the aim of the present study was to examine the role
of gender in self-image in a holistic neurorehabilitation program. Method:
Participants are a group of 48 patients ( 21 male, 27 female) with a primary
diagnosis of TBI, who participated in a 16 -week intensive holistic group pro-
gram. Patients were assessed before participation, after completing the pro-
gram and 3 years later. Assessment consisted of questionnaires regarding 
self-image. Results: The SPSS-PC program was used for statistical analysis. Multivariate analysis of variance with repeated measures showed no significant interaction effect between male and female patients on the different moments of measurement derived from scores on the Self-Image Questionnaire. Discussion and conclusions: In this study, positive changes in self-image were found, after intensive neurorehabilitation. No relationship was found between gender and changes in self-image. The determinants of successful rehabilitation need further investigation.

\section{Meta-Cognition and Brain Injury: In the Head or the Conversation?}

C. Murray ${ }^{1}$ and G. Yeates ${ }^{2}$

${ }^{1}$ Milton Keynes Neuro-Rehabilitation Team, Milton Keynes Primary Care Trust, Bucks, United Kingdom

${ }^{2}$ Community Head Injury Service, Buckinghamshire Hospitals NHS Trust, Aylesbury, Bucks, United Kingdom

$\boldsymbol{B}^{\text {ackground and aims: Meta-cognition has increasingly featured in the }}$

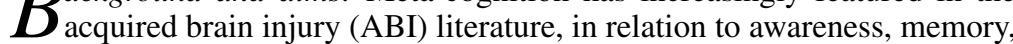
executive functioning and mood. Meta-cognitive abilities have been associated with a neural substrate in the dorso-lateral prefrontal cortex and damage to this area has been formulated to permanently undermine meta-cognitive abilities. Other conceptualisations of meta-cognitive mental processes exist, notably social psychological approaches. Method: A case is presented from couples therapy within a brain injury service of a 45-year-old survivor of a sub-arachnoid haemorrhage (affecting mid-line and right frontal structures) and his wife. Changes in his meta-cognitive abilities, ascertained from his conversational input during therapy sessions, were tracked during the first 6 months of therapy. These changes are used to provide a critical contrast between neuro-cognitive and communication/conversation models of metacognition. Results: Increased meta-cognitive ability for the survivor, with resultant improvements in the couple relationship, were achieved using the family therapy techniques of externalising and recursive/reflexive questioning. The couple referred to the haemorrhage as 'Chucky' and the survivor described a meta-cognitive stance that he increasingly used to circumvent unhelpful behaviours and interactions with his wife. Discussion and conclusions: By simply equating meta-cognitive abilities with a specific neuroanatomical structure (DLPFC), there is a danger of an infinite regress - as each reflexive stance is taken, meta-cognitive of a previous meta-cognitive process, more layers of cortex will be required, ad infinitum. Conversational frameworks describing recursive and reflexive communication offer both conceptual clarity and new invitations for both couples therapy and cognitive rehabilitation techniques and interventions. 